\title{
Adaptation of Powerline Communications-Based Smart Metering Deployments to the Requirements of Smart Grids
}

\author{
Alberto Sendin ${ }^{1, *}$, Txetxu Arzuaga ${ }^{2}$, Iker Urrutia ${ }^{1}$, Iñigo Berganza ${ }^{1}$, Ainara Fernandez ${ }^{1}$, \\ Laura Marron $^{2}$, Asier Llano ${ }^{2}$ and Aitor Arzuaga ${ }^{2}$ \\ Received: 29 August 2015; Accepted: 12 November 2015; Published: 30 November 2015 \\ Academic Editor: Thorsten Staake \\ 1 Division of Control Systems and Telecommunications, Iberdrola, Avenida San Adrian 48, 48003 Bilbao, \\ Spain; iurrutiag@iberdrola.es (I.U.); iberganza@iberdrola.es (I.B.); afernandezol@iberdrola.es (A.F.) \\ 2 Department of R \& D, ZIV-CG Automation, Parque Tecnológico de Zamudio 210, 48170 Zamudio, Spain; \\ txetxu.arzuaga@cgglobal.com (T.A.); laura.marron@cgglobal.com (L.M.); asier.llano@cgglobal.com (A.L.); \\ aitor.arzuaga@cgglobal.com (A.A.) \\ * Correspondence: asendin@iberdrola.es; Tel.: +34-94-4664787; Fax: +34-94-4664485
}

\begin{abstract}
Powerline communications (PLC)-based smart meter deployments are now a reality in many regions of the world. Although PLC elements are generally incorporated in smart meters and data concentrators, the underlying PLC network allows the integration of other smart grid services directly over it. The remote control capabilities that automation programs need and are today deployed over their medium voltage (MV) grid, can be extended to the low voltage (LV) grid through these existing PLC networks. This paper demonstrates the capabilities of narrowband high data rate (NB HDR) PLC technologies deployed over LV grids for smart metering purposes to support internet protocol internet protocol (IP) communications in the LV grid. The paper demonstrates these possibilities with the presentation of the simulation and laboratory results of IP communications over international telecommunication union: ITU-T G.9904 PLC technology, and the definition of a PLC Network Management System based on a simple network management protocol (SNMP) management information base (MIB) definition and applicable use cases.
\end{abstract}

Keywords: smart metering; smart grid; powerline communications (PLC); international telecommunication union ITU-T G.9904; distribution grid; network management system; internet protocol (IP); simple network management protocol (SNMP)

\section{Introduction}

The expansion of smart metering systems worldwide, and especially in Europe as a result of the Directive [1], Annex I.2 (last report in [2]), is now not a trend but a fact. Smart metering systems have evolved from the plain automatic meter reading (AMR) to the advanced metering infrastructure (AMI) [3], and from there, they have acquired this new name of smart metering.

Smart metering systems have common characteristics, and eventually a formal architecture [4] has been defined for them. Depending on the nature of the utility and country, parts of that system are implemented in a different way. Undoubtedly, one of the main differences among smart metering systems is the telecommunications technology used $[5,6]$. Although the different technology alternatives may be functional, they show different particularities that make the deployments different, either from the logistics, or from the investment levels, or from the operational costs. Moreover, not all of them offer the same functionality or control over the smart metering infrastructure needed for their performance. 
One of the most popular technologies for smart metering deployments is powerline communications (PLC). Among the different advantages that have been reported in literature, integration with the grid infrastructure (cables), low investment costs, no third-party intervention and good performance [7], can be mentioned. The literature also mentions some drawbacks of the technology, such as bad noise conditions in the channel, cable attenuations and some others that, in fact, are not so uncommon in other non-PLC technologies [8]. However, these drawbacks are many times just nominal, and not quantified or thoroughly tested, as in the last years, and with the advent of the so-called new generation narrowband high data rate (NB HDR)PLC technologies [9] (NB HDR PLC; international telecommunication union ITU-T G.9902 [10], ITU-T G.9903 [11], ITU-T G.9904 [12] and IEEE 1901.2 [13]) many papers have shown real results of the tens of millions of PLC smart meters deployed on the field making many of the theoretical concerns vanish. The example of the utilities in Spain is relevant, as they have deployed millions of NB HDR PLC ITU-T G.9904 systems in the last four years, solving any deployment-related difficulties, among them coupling and channel characterization aspects; the research on these topics is now part of the available products and standards, especially in low voltage (LV) grid applications. However, constant research is needed to improve the knowledge on the distribution grid and how to adapt PLC technologies to its different challenges within the smart grid domain [14-17].

There are different strategies to deploy PLC-based smart metering systems, as [18] describes. Broadband PLC (BPL) can be combined with NB HDR PLC systems in different ways. Although BPL can be deployed in LV and NB HDR PLC in medium voltage (MV), the most extended NB HDR PLC deployment scenario limits it to LV grid segments. Thus, data concentrators are deployed in secondary substations to reach the smart meters with NB HDR PLC; and these data concentrators connect to central utility systems via other technologies (non-PLC based-commercial 2G/3G/4G radio, etc.; or with BPL as [19] describes).

Smart metering systems are also mentioned profusely in the literature as the first stage towards the implementation of the smart grid [20]. The smart grid concept is a general one [21] and no single set of functionality can be found. However, it is clearly related to the integration of ICT within the electrical grid infrastructure for its better control and management. The smart grid in the distribution grid is one of the key challenges in this broad domain [22], as telecommunications are widely used to operate all primary substations [23], but are not so much spread over the secondary substations, and clearly not in LV grids, where the telecommunication technology for smart metering needs to be deployed and utilities in many occasions do not even have a precise knowledge of their LV assets and their location.

The varied functionality that is expected for a smart grid in LV is covered by the literature. First, smart metering is more or less advanced depending on the telecommunications technology used. The majority of the PLC existing systems before the new generation [24] were just able to get meter readings once in periods of days, and they cannot be considered to offer any analysis of the grid in real time [25]. Real time information is needed to optimize the operation of the grid [26] providing instantaneous information of the meters connected to the secondary substations [27]. The use of this information allows the detection of faults in the feeders (broken conductors), voltage control in the transformer, and tampering detection [28]. Reference [29] emphasizes connectivity identification (the transformer, with LV feeder and phase identification of smart meters) to allow a good customer asset management, the reduction of the technical losses, and transformer balancing. Synchrophasors are covered in [30], and reference [31] shows the possibility of remote control over LV assets, similar to that of MV levels (e.g., line protection switches). Some of these applications (feeder identification) are not feasible at a reduced cost with non-PLC technologies.

As already mentioned, LV remote control extension is assumed within the smart grid functions. MV remote control is well integrated in the electricity grid operation, although not present in all MV substations. Its purpose is to get information about the grid as well as to operate the grid elements 
(e.g., switches) remotely and safely. Remote controllable points in the LV grid will allow the same mode of operation in this LV segment of the grid.

In order to take advantage of the telecommunication infrastructure that is deployed for smart metering purposes, in the support of this smart grid functionalities including remote control, PLC is a perfect candidate. Reference [32] provides information on the feasibility of IP transport for remote control applications and the extra-bandwidth of newly deployed PLC-based networks for smart metering, and discusses smart metering as the first application of these new generation NB HDR PLC technologies. On the negative side, literature shows a lack of references to IP telecommunicated remote terminal units (RTUs) [22] over PLC-enabled LV grids, and it also declares the need to have PLC network management systems (NMSs), in order to control the performance of the telecommunication network created with PLC [33]. On the NMS side, several papers mention the need of NMS for PLC systems that are to be used for smart grid purposes [34]. However, literature references to NMS for PLC mostly focuses on BPL-related systems that were popular in the early 2000s, due to the positioning of the technology as an alternative to Internet access. Reference [35] describes a simple network management protocol (SNMP)-based (RFC 1157 and 3410) system called NEOpS for BPL over MV and LV lines. Reference [36] integrates SNMP-based BPL devices in a commercial NMS platform. Reference [37] is centered on the SNMP management information base (MIB) description.

This paper presents two novel contributions. The first one shows the multiservice capabilities of NB HDR PLC systems deployed over LV grids. The support of distribution line messaging specification/companion specification for energy metering (DLMS/COSEM) and IP applications for metering and telecontrol over a PLC network deployed initially for smart metering purposes, is simulated and tested in laboratory for the first time with a representative quantity of ITU-T G.9904 PLC network elements working in European committee for electrotechnical standardization or Comité Européen de Normalisation Électrotechnique (CENELEC) A-band, and real metering traffic. The results of these tests show the feasibility of new IP applications over existing PLC smart metering networks.

The second novel contribution of this paper is the definition of a SNMP-based NMS for NB HDR PLC systems, including a MIB definition, an interface for PLC traffic capture and the first results of a prototype implementation over a massive NB HDR PLC deployment. This NMS is fundamental to allow the effective management of telecommunication services over NB HDR PLC technologies, and this contribution if a novelty for this kind of PLC systems.

The structure of the paper beyond this introduction includes a Section 2 describing the most relevant deployments and standards of PLC-based smart metering networks. Section 3 focuses in the main difficulties of these systems to evolve into full smart grid deployments, and focuses the content of this paper on the aspects under the control of the technology or the deployments. Sections 4 and 5 focus on the feasibility of IP transport over NB HDR PLC-based smart metering networks, first with a simulation (Section 4) and then (Section 5) with real laboratory tests with ITU-T G.9904 technology. Section 6 proposes a SNMP-based NMS system for these NB HDR PLC networks, and shows the first results of a prototype implementation.

\section{Powerline Communications-Based Deployments}

PLC has been considered as a useful technology virtually since the origin of the remote metering concept itself. Since the first patents were applied for back in the beginnings of the 20th century [38], the power lines themselves have been seen as a natural medium to convey all kinds of information coming from electricity meters to remote, central locations.

Since the 1930s, a number of mainly European companies started developing ripple-control systems [39] over MV and LV networks. Although for decades these were unidirectional systems with very low data rates, the acquired experience and the progress in electronics allowed for utilities to propose a totally different kind of system in the 1980s: two way communications in the order 
of kilobits per second, to be used for close-to-real-time critical operations. In the case of MV the application was distribution automation (DA), and for LV the use of PLC for AMR gave birth to a new industry.

Based on the now withdrawn British Standard BS 6839-1 from 1987 [40], CENELEC in Europe published European standard EN 50065-1 "Signalling on low-voltage electrical installations in the frequency range $3 \mathrm{kHz}$ to $148.5 \mathrm{kHz}$. General requirements, frequency bands and electromagnetic disturbances", which in 1991 established the (narrow) frequency bands thought at the time to be enough for the development of PLC-based applications. In order to respect the existing European home system (EHS) specification which used the band from $125 \mathrm{kHz}$ to $140 \mathrm{kHz}$ with a carrier frequency of $132.5 \mathrm{kHz}, \mathrm{C}$-band was reserved for home network systems. Since also the European installation bus (EIB) specification existed in the domain of home networking at the time (and it had the option of PLC around $110 \mathrm{kHz}$ ), B-band from $95 \mathrm{kHz}$ to $125 \mathrm{kHz}$ was defined for consumer use as well, along with D-band from $140 \mathrm{kHz}$ to $148.5 \mathrm{kHz}$. A-band between $3 \mathrm{kHz}$ and $95 \mathrm{kHz}$ was left for utility applications, and it is still a very popular frequency range for PLC utility systems. EN 50065-1, in its most recent 2011 version [41], is considered a harmonized standard under the current European Union legislation.

The availability of a standard specification further spurred development efforts to obtain commercial PLC devices for remote metering. Utilities certainly saw a benefit in employing these efficient technologies to optimize metering costs while facing the challenges of deregulation in the 1990s. At an international level, there were two main standardization efforts which succeeded and set the basis for the widespread usage of PLC networks in LV metering.

The first initiative, focused on application layers, was based on the work of several utilities and industry suppliers gathered around the utility communications architecture (UCA) group, which was promoted by the American electric power research institute (EPRI) [42]. Although focused on substation automation and not in remote metering, the upper layer protocol which was adopted by UCA (the so-called manufacturing message service (MMS)) was used as the main reference for a reduced group of mainly European companies to define the simplified distribution line messaging specification (DLMS), an application layer protocol internationally standardized as part of the international electrotechnical commission (IEC) 61334 series $[43,44]$. Originally produced by IEC technical committee 57 "Power Systems management and associated information exchange" as a DA system using distribution line carrier, the industry soon looked for ways to apply it for automatic meter reading. IEC technical committee 13 "Electrical energy measurement and control" took the basis of DLMS, renamed it as device language message specification and developed an object model on top of it (an object oriented model as it was common in the 1990s) which, based on German Standard DIN 43863-3 [45], was defined as companion specification for energy metering (COSEM). The DLMS/COSEM suite was designed for any kind of transport medium, not exclusively PLC. It has been standardized as part of the IEC 62056 series, which is actively evolving since then and still provides the application layer for many AMR PLC systems.

The second effort also crystalized as part of the IEC 61334 series, and it mainly dealt with the lower layers (physical layer (PHY) and media access control (MAC)) of the PLC stack. Since the 1980s, a number of bidirectional PLC systems had been designed and produced for both MV and LV grids, usually for frequencies below $500 \mathrm{kHz}$. Five of them ended up being standardized as Part 5 "lower layer profiles" of IEC 61334. Reference [46] specified the spread frequency shift keying (S-FSK) modulation by Landis + Gyr, with two carriers set apart $>10 \mathrm{kHz}$ which are FSK modulated for data rates multiple of $0.3 \mathrm{kbps}$ in LV. Reference [47] defines a binary FSK modulation proposed by ENEL (Rome, Italy), with a carrier at $82.05 \mathrm{kHz}$ and $0.6 \mathrm{kbps}$ in the case of $\mathrm{LV}$, and a carrier at $72 \mathrm{kHz}$ and $1.2 \mathrm{kbps}$ in the case of MV. Reference [48] by Adaptive Networks describes a direct sequence spread spectrum adaptive wideband (SS-AW) modulation for LV, while [49,50] represent systems developed by $\mathrm{ABB}$, an implementation of multi-carrier modulation (MCM) with differential PSK for MV and spread spectrum fast frequency hopping (SS-FFH) for LV, respectively. 
Of the above, DLMS/COSEM over S-FSK systems were the most commonly adopted deployment option. The exception worthwhile mentioning is the massive FSK technology deployment by utility ENEL in Italy, which used a proprietary application layer.

Since approximately 2005 [51], the advancement of semiconductor large-scale integration and the increasing bandwidth needs of real-time DA and smart metering facilitated the appearance of a new generation of PLC systems. Also the experience of several utilities with BPL networks in the $2-30 \mathrm{MHz}$ frequency range led to the application of traditionally broadband techniques (e.g., orthogonal frequency division multiplexing (OFDM), adaptive modulation, coding and interleaving) to PLC systems below $500 \mathrm{kHz}$ for distribution networks.

With regards to the IEC 61334 legacy PHY layers discussed above, the new generation of NB HDR PLC in the A-band and above provides several advantages: use of the full available bandwidth instead of a couple discrete frequencies (avoiding inter carrier interference (ICI) thanks to OFDM techniques), tolerance against frequency selective PLC channels, much higher data rates (up to $1 \mathrm{Mbps}$ in the case of [52]), easier channel equalization, robustness to narrowband interference and even a more cost-effective design thanks to the relative simplicity of OFDM implementation in currently available processors.

There are currently numerous examples of narrowband PLC deployment for smart metering throughout the world. ITU-T G.9904 (PRIME) is a technology currently deployed in some seven million smart meters in Australia, Korea, Latvia, Poland, Portugal, Spain, etc. Other technologies are deployed or in the process of being deployed in Austria, Belgium, Brazil, China, Denmark, Finland, France, India, Italy, Japan, Luxemburg, Sweden, etc. In Europe, approximately $90 \%$ of the currently installed smart meters employ PLC and the expectation is that by 2020 just one third of the smart meter deployments will use other technologies (mostly public cellular networks) [53].

\section{Smart Grid beyond Smart Metering in Powerline Communications-Based Systems}

As it was already mentioned, smart metering is often the starting point of the smart grid [22]. With this approach, smart metering is one of the applications running over the telecommunications infrastructure that would carry the different smart grid services (smart metering being one of them). Utilities involved in smart meter rollouts, realize that rather than deploying a smart metering infrastructure, they are deploying telecommunication networks. These PLC networks provide a PLC-based "coverage" area, linked to the LV grid that is able to support additional services and applications apart from the feature of managing smart meters. Other smart grid related applications, such as distributed energy resources (DER) integration and electric vehicle (EV)-related ones, may use the same telecommunication network as well.

PLC technologies are seen as the preferred option to provide the data services required by the new applications, as they provide coverage through the pervasive LV grid. NB HDR PLC technologies are multiservice by design. However, the systems in which they are included may or may not reflect this capability. These systems need to be prepared to support different data services with varying requirements in terms of quality of service (QoS; i.e., throughput, latency and jitter limits) depending on the smart grid functionality they need to support.

After a thorough review of existing PLC networks deployments in Europe where the most relevant PLC for smart metering deployments exist [2], two main obstacles can be identified with PLC technologies. The first obstacle is related to network management. PLC networks, like any other telecommunication infrastructure, need to be managed. It is critical that the PLC network operator collects real-time information related to its performance. This is the only way to control and be able to improve the operation of a telecommunication network, and to guarantee the availability of its data services.

The second obstacle is related to the assurance of the PLC network available data capacity. Utility use of the spectrum in LV power lines in Europe is limited to A-band (between $3 \mathrm{kHz}$ and $95 \mathrm{kHz}$ ). Several LV channel characterizations [54] show that the lower part of the band is useless. This is 
the reason why most PLC systems use the upper part of the band (from $30 \mathrm{kHz}$ or $40 \mathrm{kHz}$ ). Thus maximum capacity is restricted by the limited bandwidth of $40 \mathrm{kHz}$ and by the noises of different nature that can be found in the band [55]. As certain smart grid applications (e.g., remote control) can be jitter-sensitive [22], PLC systems need to provide resource reservation mechanisms to deliver QoS service-levels, for example, to remote control data flows. This obstacle will be solved with the existing features of NB HDR PLC standards (e.g., the use of the contention free period (CFP) of the ITU-T G.9904 standard) that however and logically cannot prevent electricity grid noises. Regarding the frequency band enlargement, it is important to mention that in America and Asia, utilities can use frequencies beyond $95 \mathrm{kHz}$. In the USA, according to the Federal Communications Commission FCC Part 15 under Title 47 of the code of federal regulations, the band can be extended from $10 \mathrm{kHz}$ to $490 \mathrm{kHz}$ [9]. In the case of Japan, utilities are allowed to use telecommunication equipment in frequencies between $10 \mathrm{kHz}$ and $450 \mathrm{kHz}$ (association of radio industries and businesses standard ARIB STD T-84) [9]. South Korea is currently using International Organization for Standardization ISO/IEC 12139-1 [56] with Korean Standard KN60 defined limits for frequencies below $500 \mathrm{kHz}$. This extended frequency band, with an appropriate regulation in the compatibility levels, is highly beneficial for the development of telecommunication networks for smart grid applications over PLC. As stated in [57] for Europe, emission levels of PLC systems need to comply with EN 50065-1. Unfortunately, there are no standards that define the compatibility levels in CENELEC A-Band, which may impact negatively on the communications quality due to not intentional disturbances produced by devices connected to the electricity grid exclusively for power applications (power inverters as studied in $[58,59]$ and other electronic devices).

\section{A Capacity Assessment of Existing Narrowband Powerline Communications Resources in the CENELEC A-Band}

A simulation of a simple but representative scenario of ITU-T G.9904 technology is developed in this section in order to make a capacity assessment of NB HDR PLC networks in the CENELEC A-band. ITU-T G.9904 NB HDR PLC system is the most extended and deployed PLC system of all the standards available.

This PLC network simulator covers different network elements and traffic conditions, in a smart metering data transport scenario. The objective of the simulation is the estimation of the maximum capacity of NB HDR PLC systems for IP data transmission in the presence of smart metering traffic.

The simulation consists of a $C$ language coded software that implements the carrier sense multiple access collision avoidance (CSMA/CA) algorithm in ITU-T G.9904 for the smart metering profile (maximum raw speed of $20 \mathrm{kbps}$ at PHY) for multiple nodes in a single collision domain. The software is coded to emulate different traffic patterns, all of them in the worst case of collision domain sharing. The results are extracted based on frame error rate (FER), a way to measure the packet loss probability. Application level rate is calculated based on FER, including retries (automatic retransmission request: ARQ).

The simulated network topology is composed of one base node (BN), a variable number of service nodes (SNs) associated to the smart metering (they do not exchange IP traffic), and one SN (device under test: DUT,) sending IP traffic over the PLC network. Other assumptions are:

- The BN reaches all SNs without "switching" (signal repetition) in one single collision domain.

- There is no external noise source.

- Network traffic is based on a Poisson distribution with a mean time obtained from real field scenarios. The transmission attempt follows this distribution; note that repetitions (if needed) will follow CSMA/CA algorithm.

The time distribution parameters considered per traffic type in this simulation are: 
- Control traffic for the BN. It follows a Poisson distribution with a mean of $0.5 \mathrm{~s}$ for networks larger than 32 nodes and $16 / \mathrm{N}$ s where $\mathrm{N}$ is the number of nodes, for smaller networks (the difference is made to keep a stable control traffic in the subnetwork).

- Control traffic for each SN. It follows a Poisson distribution with a mean of N/2 s for networks larger than 32 nodes, and $16 \mathrm{~s}$ for smaller networks.

- IP traffic for the SN under test (the DUT) and the BN. It follows a Poisson distribution with a mean of $10 \mathrm{~ms}$, for the first transmission attempt. Following ARQ retransmissions will have a mean of $1 \mathrm{~s}$. The ARQ timeout is $1 \mathrm{~s}$.

- Smart metering application data for each SN. It follows a Poisson distribution with a mean of $1.6 \mathrm{~s}$.

These traffic patterns show the following PHY protocol data unit (PPDU) length:

- Control traffic includes three symbols of payload.

- Data traffic (common to IP and smart metering traffic) includes a 14 symbols payload.

The different simulation scenarios consider the following aspects:

- PLC network size (different number of SNs).

- Traffic source:

- Downlink. The traffic sent from the BN.

- Uplink. The traffic sent from the SN-DUT.

- Traffic conditions in the PLC network:

- Transmission control protocol (TCP) transmission of the DUT without smart metering data (PLC control traffic of the SNs only).

- TCP transmission of the DUT with intensive smart metering data.

Figure 1 shows the FER evolution as the PLC network size increases (SNs in abscissa axis). Figure 2 shows the IP data rate (kbps) of the DUT, both uplink and downlink, as the number of SNs grows.

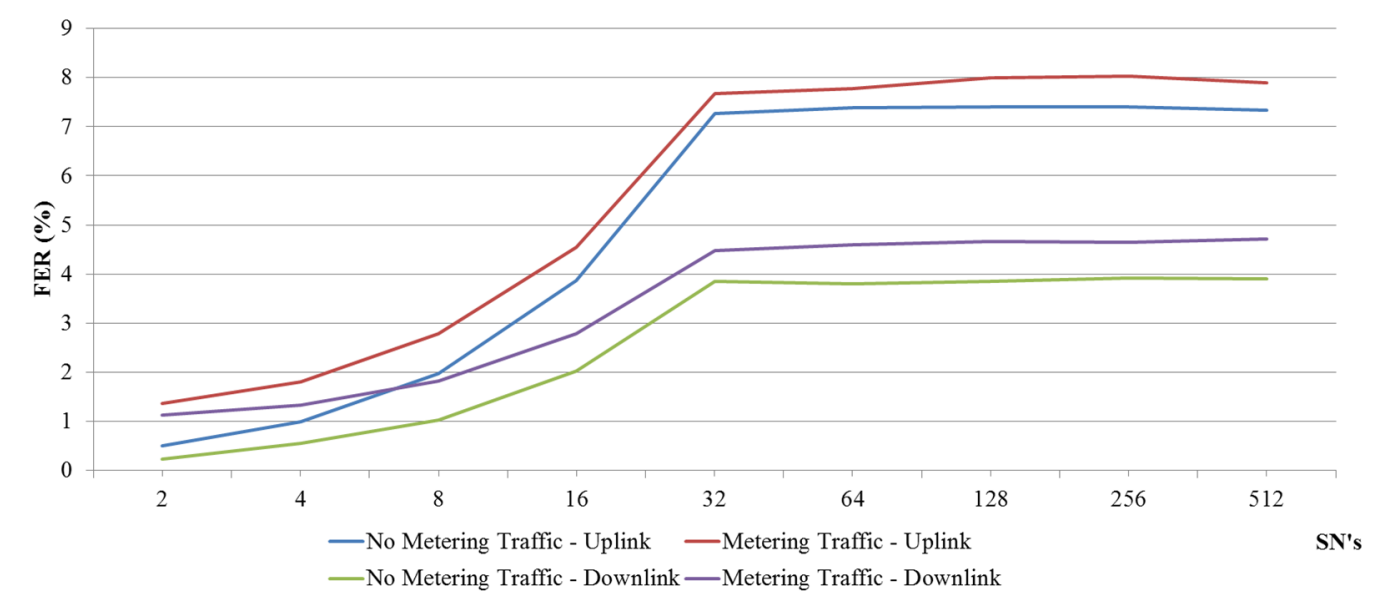

Figure 1. Frame error rate (FER) simulation based on ITU-T G.9904. International telecommunication union: ITU. 


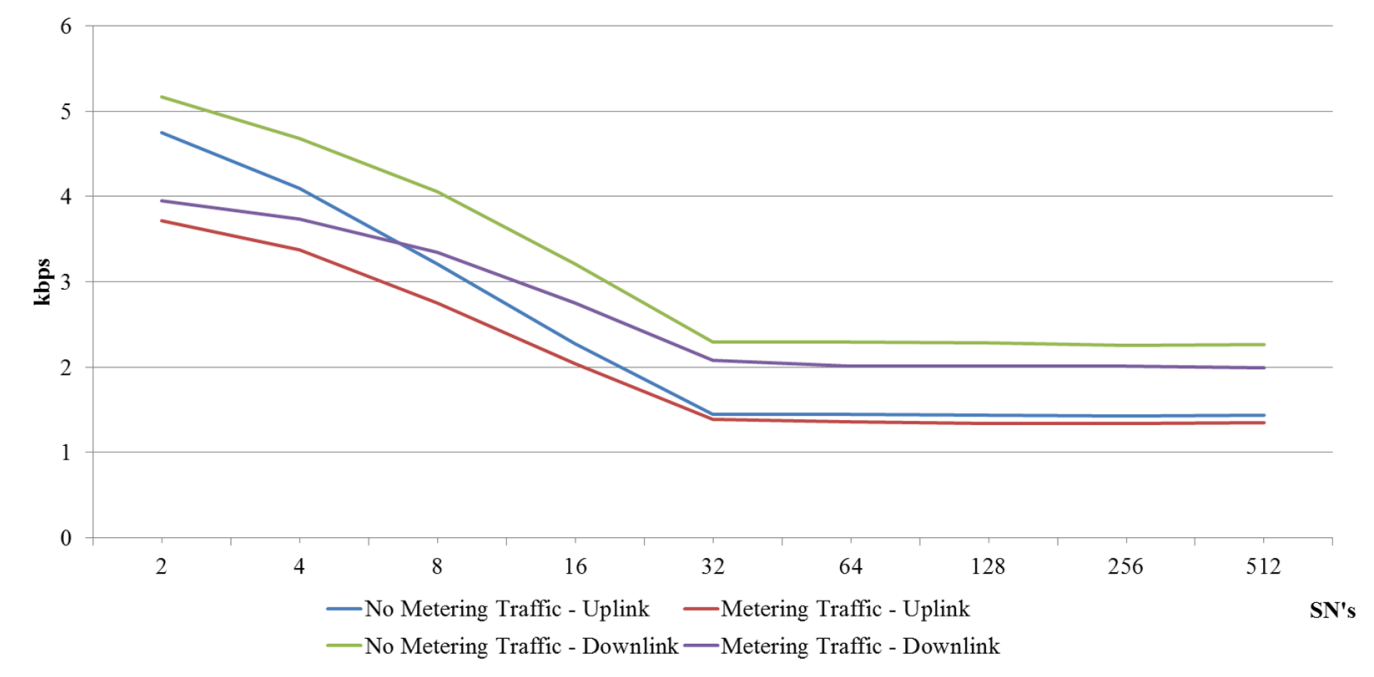

Figure 2. Data rate simulation based on ITU-T G.9904.

\section{Internet Protocol over Narrowband High Data Rate Powerline Communications in CENELEC A-Band}

ITU-T G.9904 PLC builds a multiservice network designed to support multiple applications. Different convergence layers (CSs) are defined in order to optimize each data type transport over PLC.

- 432-CS (for IEC 61334-4-32) is oriented to DLMS/COSEM smart metering applications.

- Internet protocol-convergence sublayer (IP-CS) enables the use of this multiservice network for other technologies over IP.

Figure 3 shows the versatility of ITU-T G.9904 NB HDR PLC network. Smart meters' data is collected over PLC while simultaneously IP access to other applications is allowed.

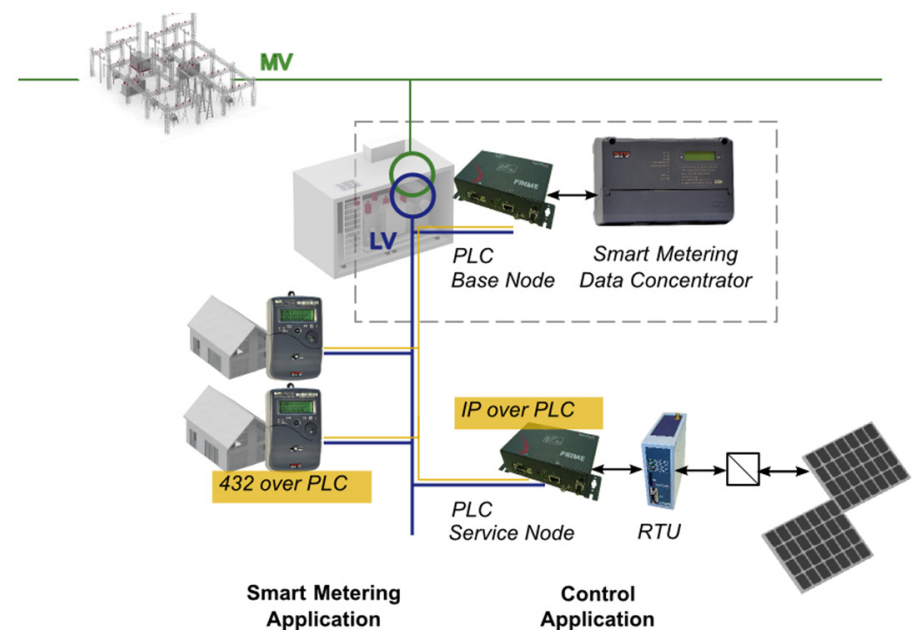

Figure 3. Multiservice network built over ITU-T G.9904.

\subsection{Support for Multiservice Internet Protocol Profile (Internet Protocol-Convergence Sublayer: IP-CS)}

This section analyzes the traffic to be exchanged over PLC to make the multiservice network possible. Note that ITU-T G.9904 NB HDR PLC BN is ready to manage different communication profiles, smart metering profile (over 432-CS) and any other application running over IP (over IP-CS) will be described. 
The MAC registration process is independent from the profile used. It is initiated from the $\mathrm{SN}$ that automatically sends a registration request control frame (REG_REQ) when it is aware of the presence of a network (notified by the master of the network, BN). The unique BN of the network acknowledges this registration process and accepts the required capabilities for this $\mathrm{SN}$ (REG_RSP). Final confirmation is sent from the SN (REG_ACK).

Following is a real ITU-T G.9904 PLC MAC level capture using the ZIV PRIME Manager tool [60] showing the registration process of a SN. This node has an EUI-48 address of $40: 40: 22: 02: 4 \mathrm{f}: \mathrm{b} 1$ and after this registration process it has been assigned a local node identifier lnid:2987. The first lines are metadata information, and the real hexadecimal transmitted content is included in the 16 bytes rows bellow, behind the "I" sign.

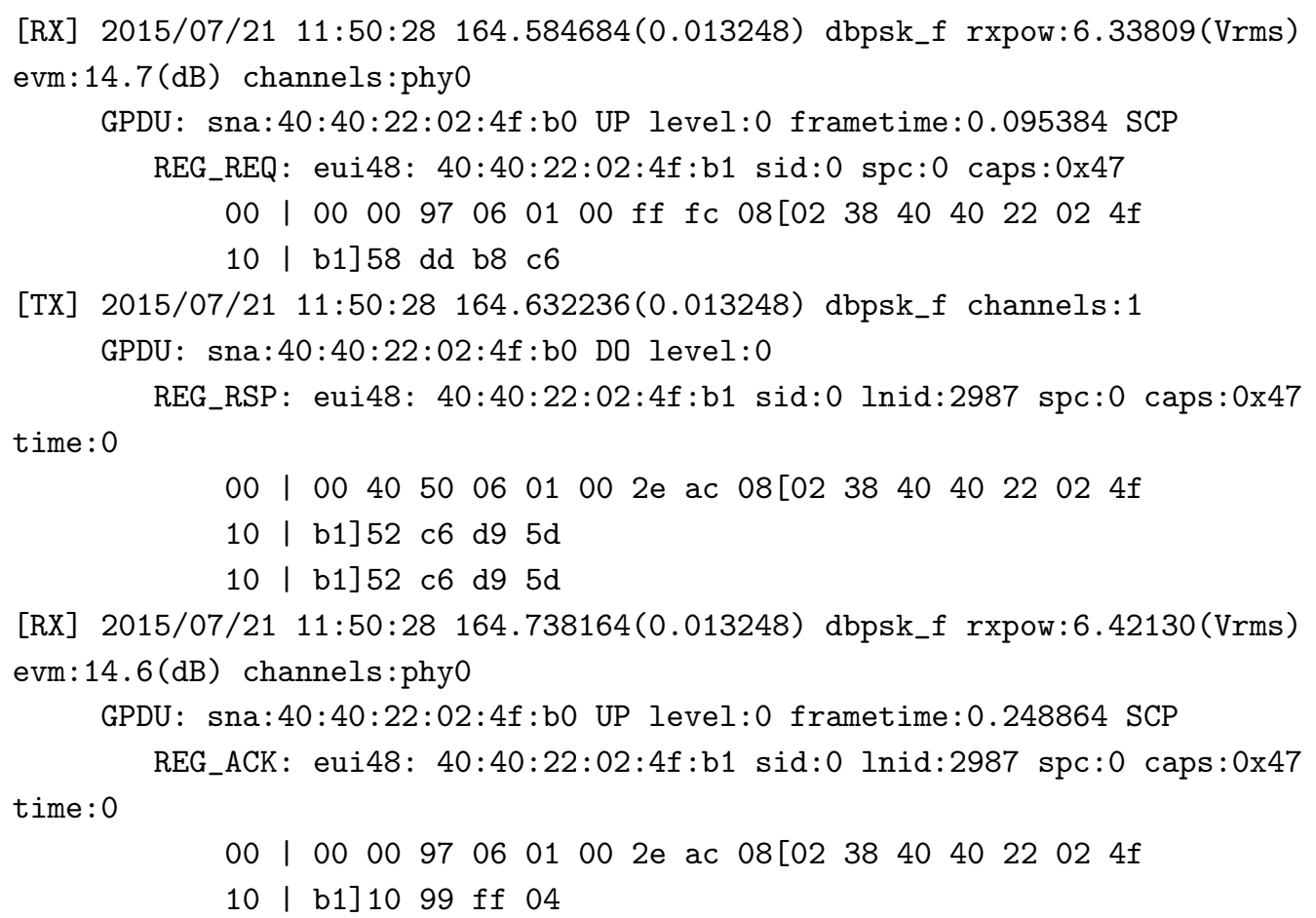

Once the registration is complete, a profile dependent connection is opened from the SN. This is an example of multiservice IP profile establishment; connection is requested from the SN with a control message (CON_REQ_S) and confirmed from the BN (CON_REQ_B).

- The BN maintains a database of IP addresses and EUI-48 addresses. Address resolution then operates by querying this database. A SN must establish a connection to the address resolution service running on the $B N$, using the connection type TYPE_CL_IPv4_AR (type:1). Marked in green in the example below.

- For packets to be transferred, a connection needs to be established between source and destination nodes. IP-CS will examine each IP packet to determine the destination EUI-48 address and therefore a MAC connection is established to the remote device. The TYPE value of this connection is TYPE_CL_IPv4_UNICAST (type:2). Marked in blue in the example below.

- Inside the data included in the request message, the local IP address is provided so that the remote device can add this new connection to its cache of connections for sending data in the opposite direction.

- The use of Van Jacobson Header Compression (RFC 1144) is also negotiated as part of the connection establishment. 


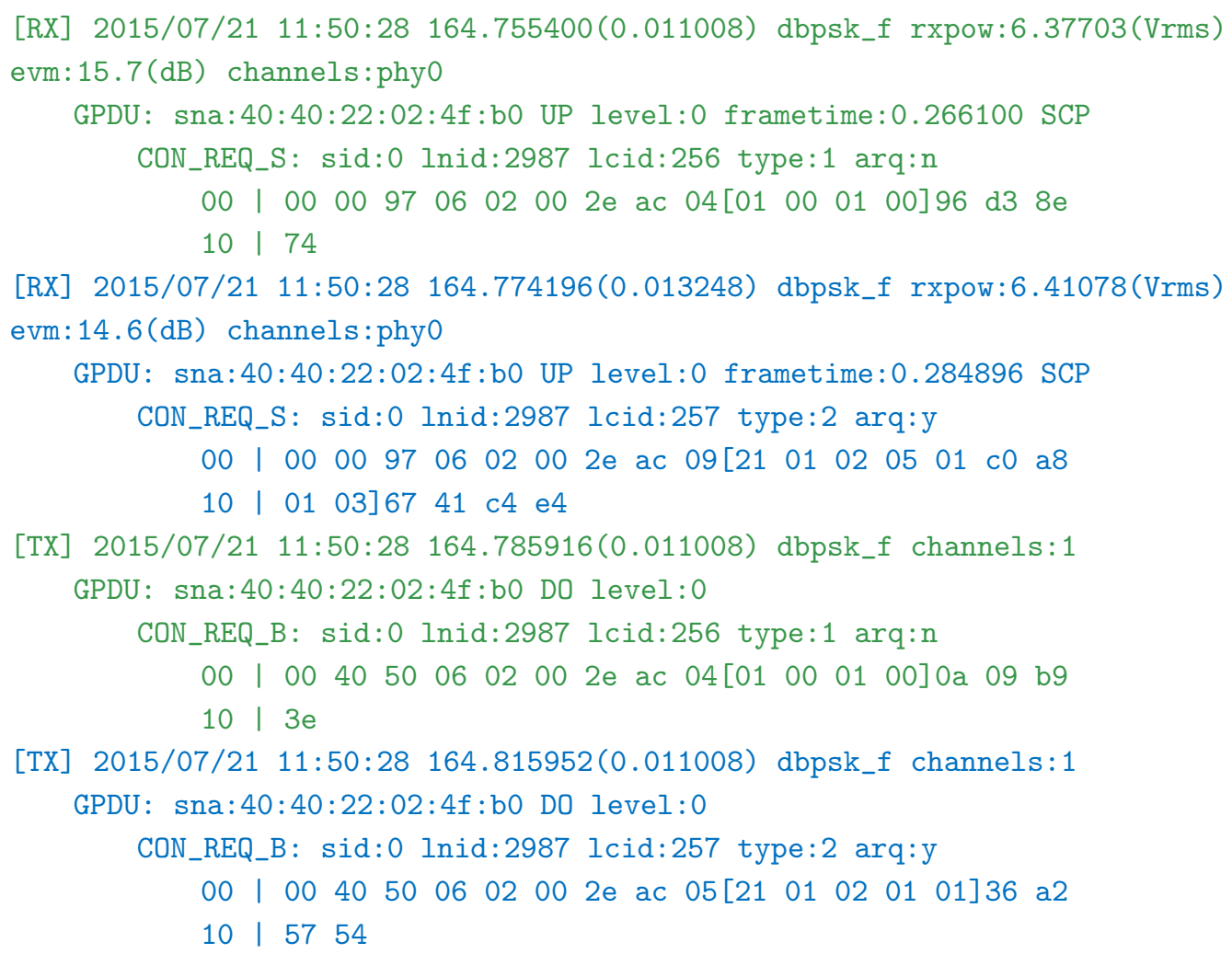

Once registration is achieved and the IP-CS connection is established, IP packets can be sent over PLC. Following is a capture of internet control message protocol (ICMP) packets being exchanged over IP with this same node that had been assigned a local node identifier lnid:2987. Note that once the connection is ready, IP packets sent over PLC will be shown as DATA packets in the MAC layer. This DATA is sent over the TYPE_CL_IPv4_UNICAST connection that was assigned a connection identifier of Icid:257.

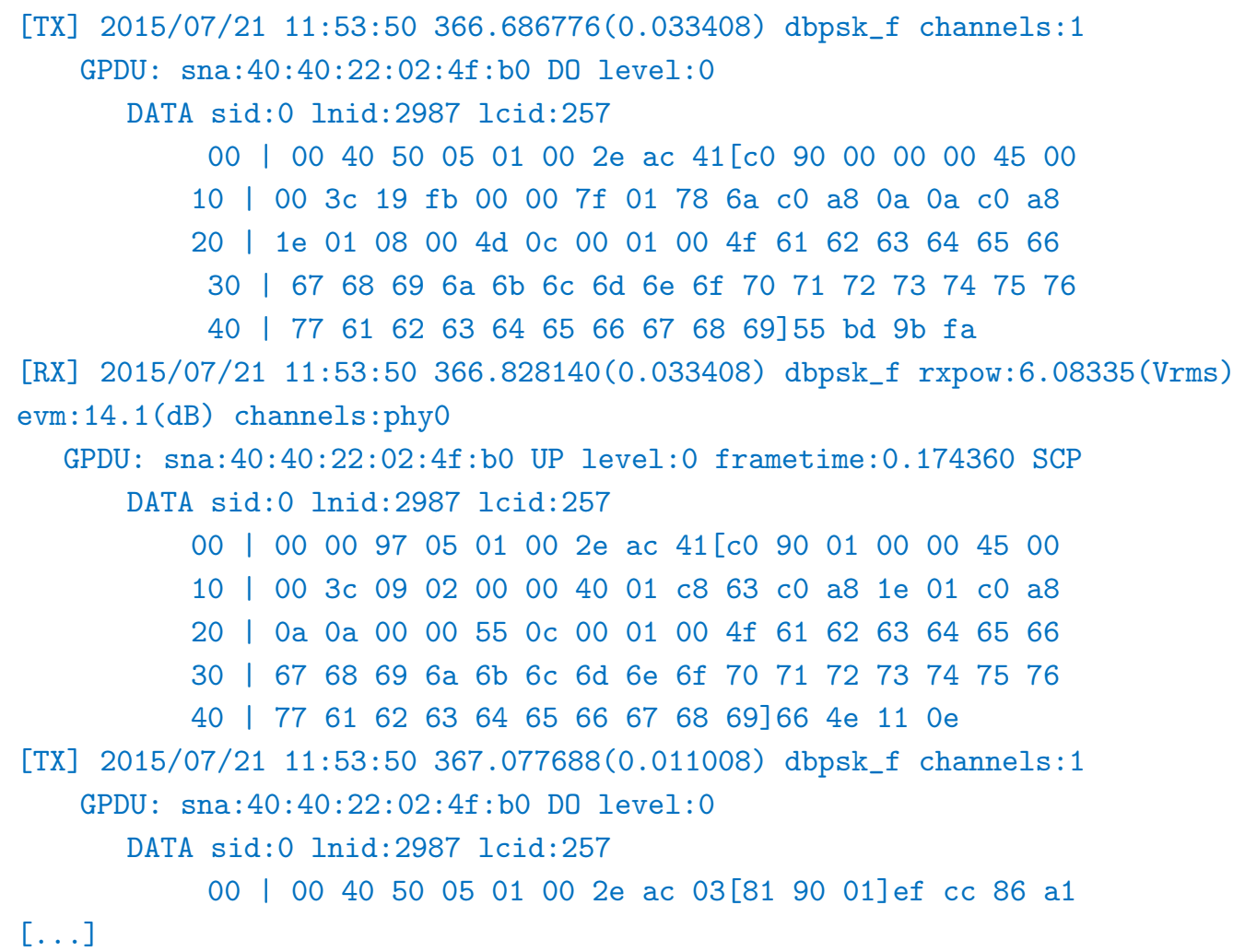


As described above, ITU-T G.9904 NB HDR PLC builds a multiservice application so different data profiles can be exchanged simultaneously. The following traffic capture shows an example of this capability. This MAC capture includes the traffic of a network where a smart meter is being read while simultaneously ICMP packets over IP are being exchanged.

- Smart meter with serial ZIV0041213078 with EUI-48 40:40:22:74:dc:96 and lnid:11162 details are being read using 432-CS. DLMS traffic is sent over TYPE_CL_432 connection (type:3) that was assigned a connection identifier lcid:258. Marked in red in the example below.

- At the same time ICMP packets (ping) are being exchanged with SN of PLC IP 192.168.1.3 with MAC $40: 40: 22: 02: 4 f: b 1$ and lnid:2987. IP traffic is sent over TYPE_CL_IPv4_UNICAST connection that was assigned a connection identifier lcid:257. Marked in blue in the example below.

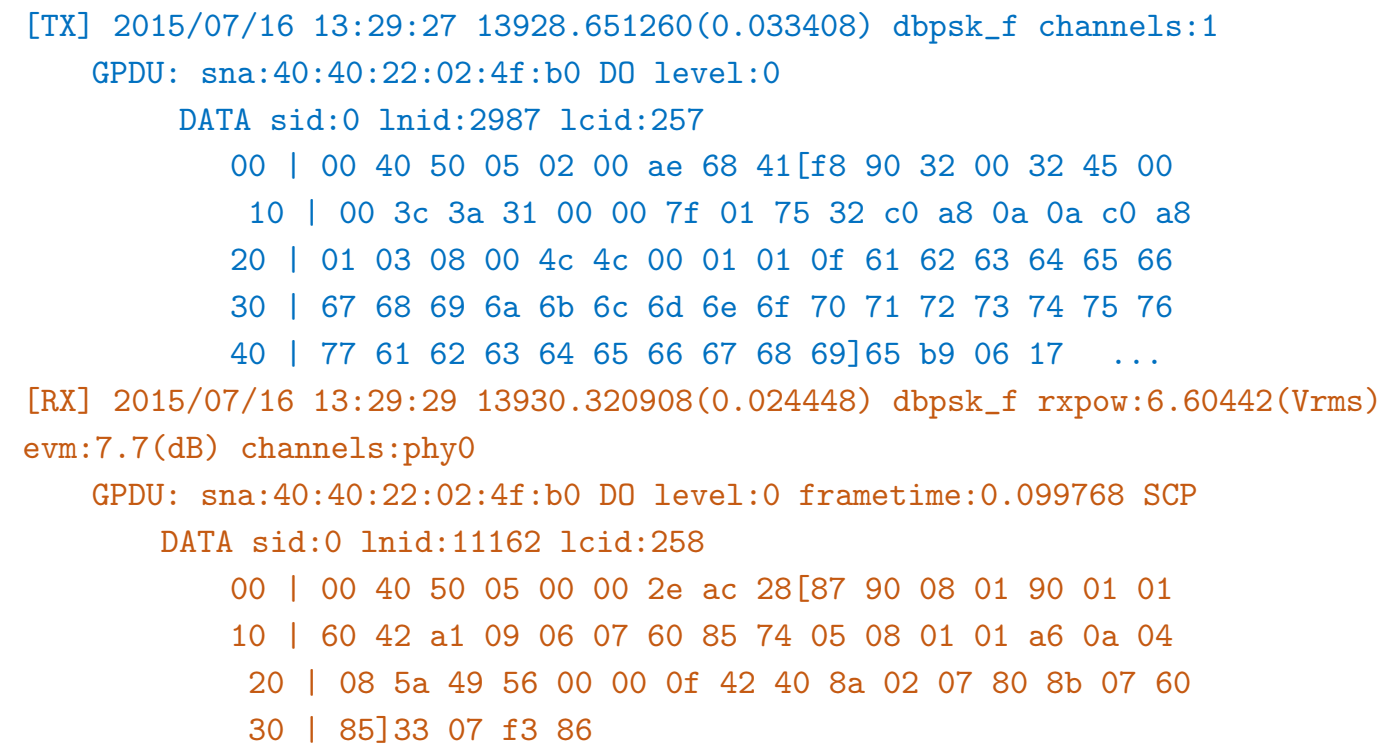

5.2. Internet Protocol Over Narrowband High Data Rate Powerline Communications Tests in Non-Switched Environments

\subsubsection{Test Description}

This test plan has been defined in order to determine the performance and best possibilities of IP transport in flat PLC networks, where no switching levels exist (i.e., no signal repetition is needed in the network).

The following incremental steps are defined in order to validate these two scenarios:

- Multiservice IP traffic exchange over PLC channel.

- Validation within the same PLC network of simultaneous smart metering (DLMS over 432-CS) and multipurpose IP traffic connections.

Figure 4 shows the test setup used for the validation. For each application, the following devices are involved:

- DLMS smart metering application:

- Smart metering data concentrator.

- Six smart meters.

- Multiservice application over IP:

- 2 SNs implementing IP-CS. 
- In order to manage the setup, a BN that supports both profiles is required. The BN is a ZIV PBN [61].

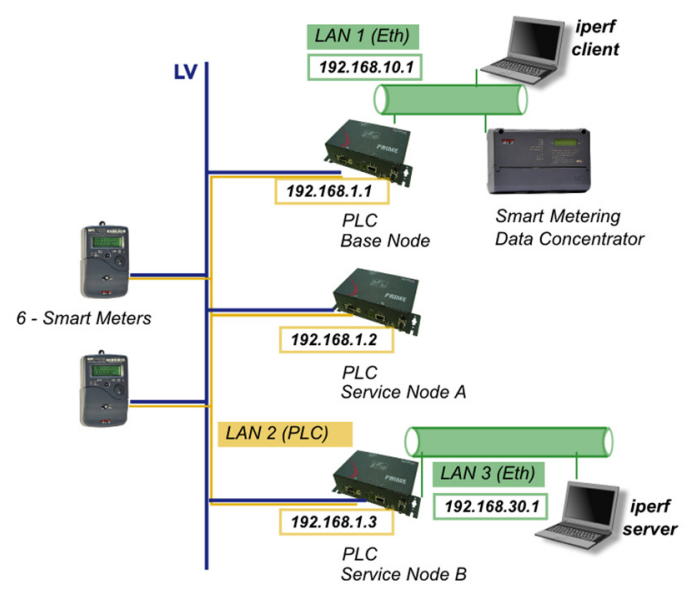

Figure 4. Multiservice network built over ITU-T G.9904 narrowband high data rate (NB HDR) powerline communications (PLC). Test setup.

Measurements are taken under different traffic conditions:

(1) Basic connectivity ICMP tests (ping, as per RFC 792 and RFC 1122).

(2) Performance measurements of TCP/IP traffic over PLC (using the iperf tool [62] in a client-server setup).

a TCP/IP traffic without smart metering data (PLC control traffic of the nodes)-Minimum network size of two nodes (see Section 5.2.2a).

b TCP/IP traffic without smart metering data (PLC control traffic of the nodes)-Medium network size of eight nodes (see Section 5.2.2b).

c TCP/IP traffic with intensive smart metering data-Medium network size eight nodes (see Section 5.2.2c).

\subsubsection{Tests Results}

Results obtained with each test setup scenario and traffic conditions are described in this section.

(1) Basic connectivity ICMP tests (ping).

Connectivity is validated between the different local area networks (LANs) shown in Figure 4.

First connectivity step is between LAN1 (Ethernet) and LAN2 (PLC). ICMP packets of 32 bytes are sent, with an average time of $214 \mathrm{~ms}$. All ICMP packets sent are successfully received ( $0 \%$ packet loss at this level).

Second connectivity step involves LAN1 (Ethernet) and LAN3 (Ethernet), making the transport through LAN2 (PLC). This step is covered in the performance measurements described below.

(2) Performance measurements using iperf tool of TCP/IP traffic over PLC.

Different tests are performed modifying these TCP parameters:

- TCP window size (socket buffer size)—both at client and server side.

- The best results are obtained with default (adaptive) value, although a window of 300 bytes offers similar performance during this test.

- The bigger the window fixed (above this value), the lower the performance. 
- TCP maximum segment size (maximum transmission unit (MTU)).

- Best results are obtained with default value, i.e., 1500 bytes.

The results shown are those obtained with the default TCP window size and MTU selection.

a TCP/IP traffic without smart metering data (PLC control traffic of the nodes only)_Minimum network size.

- PLC network size: two nodes.

- MAC mechanism: according to ITU-T G.9904 specification.

- Traffic conditions: TCP transmission of the DUT without smart metering data. This step is measured between different network elements (BN-SN, and SN-SN), so both scenarios results are shown below. SNA is SN A and SNB is SN B.

\section{Test scenario 1: End nodes BN-SNB}

In this scenario traffic measured is:

- Downlink traffic, BN as transmitter (iperf client) and SNB as receiver (iperf server).

Iperf started at the client side on LAN1 (Ethernet).

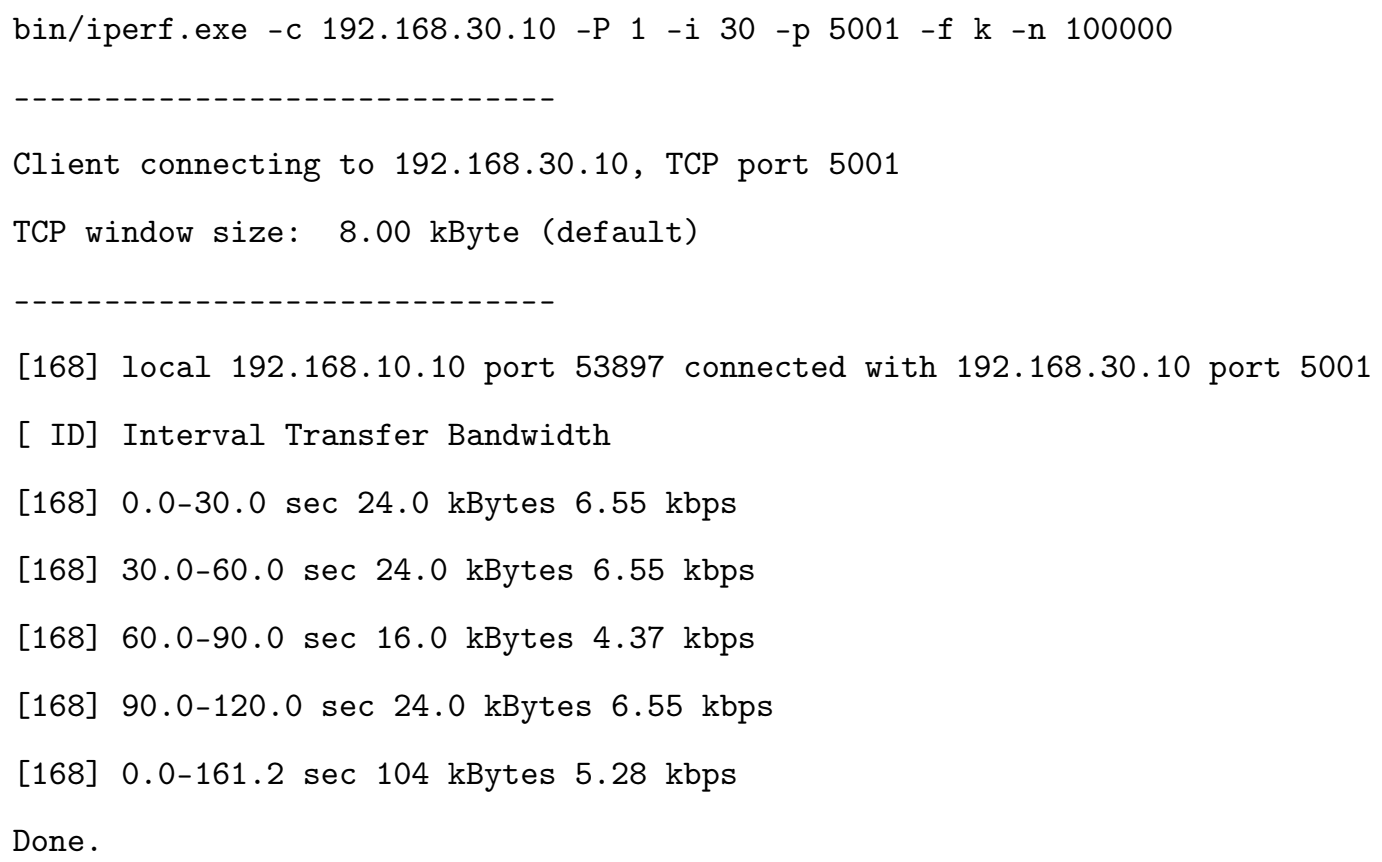

At the server side at LAN3 (Ethernet) the following results are shown. These values show different test executions performed under these same conditions.

local 192.168.30.10 port 5001 connected with 192.168 .10 .10 port 53897

First iteration result:

0.0-160.9 sec 104 kBytes 5.29 kbps

Second iteration result:

0.0-170.2 sec 104 kBytes $5.00 \mathrm{kbps}$

Third iteration result:

0.0-189.4 sec 104 kBytes $4.50 \mathrm{kbps}$

Test scenario 2: End nodes SNA-SNB

Iperf started at the client side at the LAN (Ethernet) behind SNA. Therefore client location is different from the test scenario described above. 
- This kind of traffic was not described in the simulation process. SNA as transmitter (iperf client) and SNB as receiver (iperf server).

At the server side at LAN3 (Ethernet) the following results are shown. These values show different test executions performed under these same conditions.

First iteration result:

$$
0.0-559.4 \mathrm{sec} 104 \mathrm{kBytes} 1.52 \mathrm{kbps}
$$

Second iteration result:

$0.0-460.9 \mathrm{sec} 104$ kBytes $1.85 \mathrm{kbps}$

Third iteration result:

$0.0-494.6 \mathrm{sec} 104 \mathrm{kBytes} 1.72 \mathrm{kbps}$

b TCP/IP traffic without smart metering data (PLC control traffic of the nodes only)—Medium network size.

- PLC network size: eight nodes.

- MAC mechanism: according to ITU-T G.9904 specification.

- Downlink traffic, BN as transmitter (iperf client) and SNB as receiver (iperf server).

- Traffic conditions: TCP transmission of the DUT without smart metering data.

At the server side at LAN3 (Ethernet) the following results are shown. These values show different test executions performed under these same conditions.

First iteration result:

$0.0-197.4 \mathrm{sec} 104 \mathrm{kBytes} 4.32 \mathrm{kbps}$

Second iteration result:

$0.0-209.8 \mathrm{sec} 104$ kBytes $4.06 \mathrm{kbps}$

c TCP/IP traffic with intensive smart metering data-Medium network size.

- PLC network size: eight SNs.

- MAC mechanism: according to PLC PRIME 1.3.6 specification.

- Downlink traffic, BN as transmitter (iperf client) and SNB as receiver (iperf server).

- Traffic conditions: TCP transmission of the DUT with intensive smart metering data.

At the server side at LAN3 (Ethernet) the following results are shown.These values show different test executions performed under these same conditions.

First iteration result:

$$
0.0-227.2 \text { sec } 104 \text { kBytes } 3.75 \mathrm{kbps}
$$

Second iteration result:

$0.0-257.3 \mathrm{sec} 104 \mathrm{kBytes} 3.31 \mathrm{kbps}$

Third iteration result:

$0.0-243.9 \mathrm{sec} 104 \mathrm{kBytes} 3.49 \mathrm{kbps}$

Table 1. Summarizes performance measurements obtained during the tests.

Table 1. IP over narrowband high data rate (NB HDR) powerline communications (PLC) performance measurements. Service Nodes: SNs; TCP: transmission control protocol; IP: Internet protocol.

\begin{tabular}{cccc}
\hline Traffic type & Traffic conditions & Number of SNs & Data rate (kbps) \\
\hline Downlink & TCP/IP with PLC control traffic only & 2 & $4.50-5.29$ \\
Downlink & TCP/IP with PLC control traffic only & 8 & $4.06-4.32$ \\
Downlink & TCP/IP with intensive smartmetering traffic & 8 & $3.31-3.75$ \\
Between two SNs & TCP/IP with PLC control traffic only & 2 & $1.52-1.85$ \\
\hline
\end{tabular}




\subsubsection{Test Results Analysis and Conclusions}

The application level data rates summarized in Table 1 confirm the simulation prepared for the capacity assessment of NB HDR PLC networks in CENELEC A-band. Figure 2 shown as part of the simulations is repeated below in Figure 5, highlighting the conditions validated in the test environment.

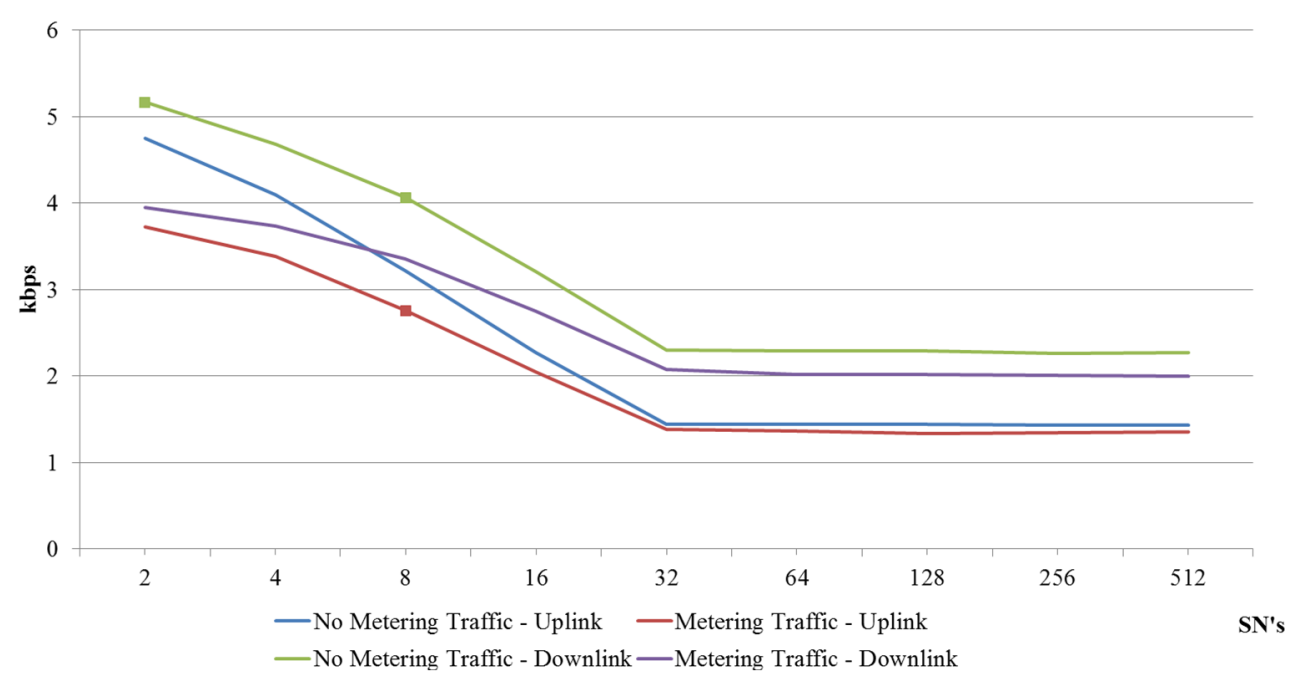

Figure 5. Simulation results validated in the tests plan based on ITU-T G.9904.

FER (\%) and data rate (kbps) obtained in the simulation are therefore a valid assessment of the existing extra-capacity in NB HDR PLC technologies, when smart metering traffic is present (this profile, with binary phase shift keying (BPSK) modulation and forward error correction (FEC) provides a maximum of $20 \mathrm{kbps}$ raw PHY speed).

\subsection{Internet Protocol over Narrowband High Data Rate Powerline Communications Tests in} Switched Environments

\subsubsection{Test Description}

As it has been stated in some references to ITU-T G.9904 technology [12], PLC signal repetition is one of the more important features of NB HDR PLC systems to overcome the effects of noise.

Further tests have been carried out in realistic environments such as the one referenced in [63] without any noise filtering, with larger quantities of SNs and several switching (repetition) levels, to understand the effect of switches in the IP data rates.

The test scenario consists of one $\mathrm{BN}$ and $50 \mathrm{SNs}$ connected simulating four meter-room concentrations. The data concentrator connected to the BN is a ZIV device [61], and it has been used to create the application level metering traffic. The smart meters used are devices from the vendors ZIV, Landis + Gyr, Sagemcom, Orbis and Sogecam, as referenced in [64,65]. The configuration of the PLC network follows the smart metering profile [64] defined for the technology, with ARQ, lowest throughput modulation (i.e., DBPSK with error correction) and 64 byte word Segmentation and Reassembly (SAR) to minimize the effect of noise.

The PLC network topology that has been recorded is shown in Figure 6. The SN used as DUT to communicate through IP with the BN, has been connected in different positions of the topology, as Figure 6 highlights in blue color. 

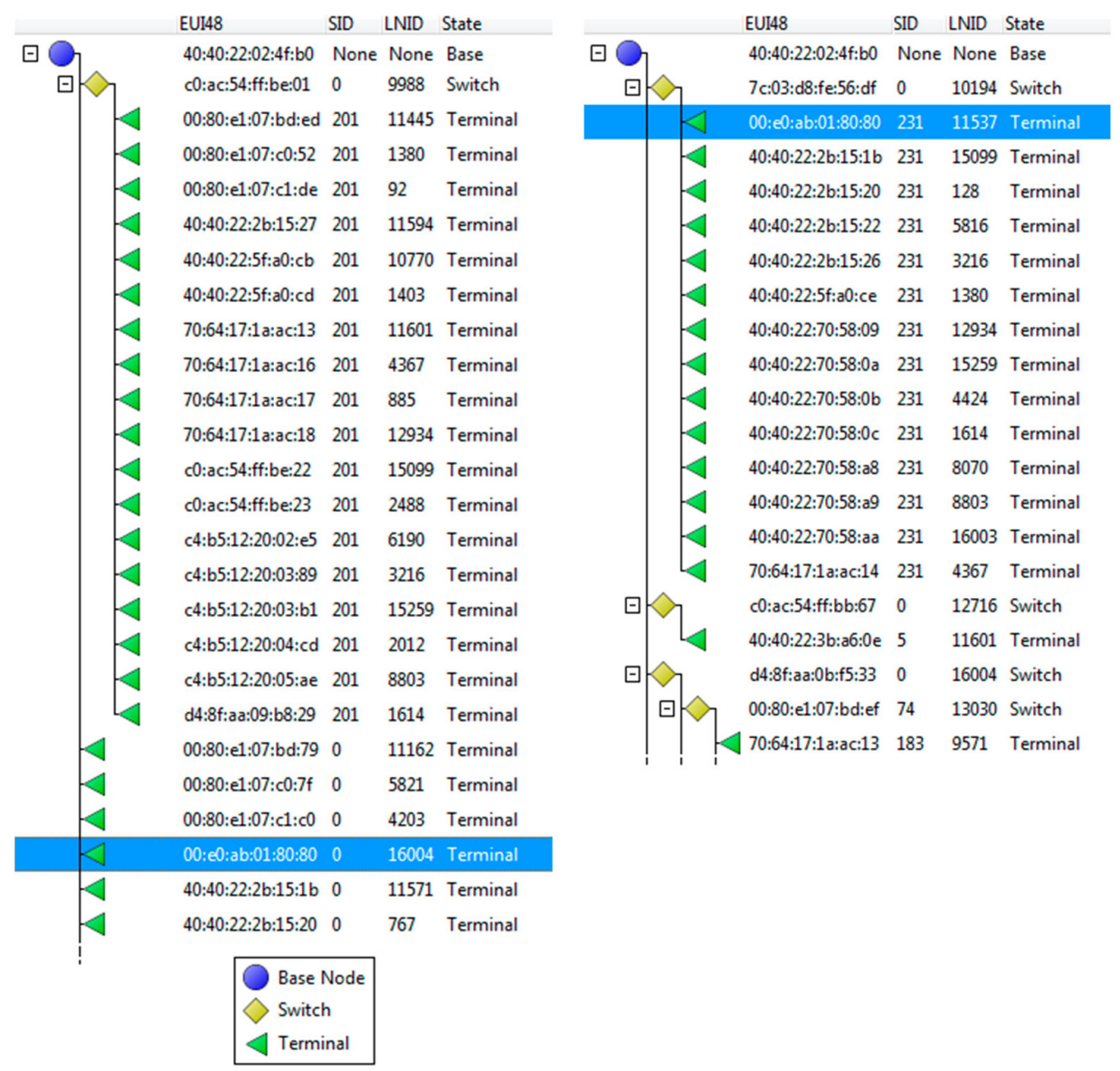

Figure 6. Topologies (first level on the left and second level on the right) for switching environment tests.

\subsubsection{Tests Results}

ICMP pings have been used to evaluate the performance of the IP connectivity. 32 bytes and 64 bytes of payload have been sent from the BN to the SN used as DUT (150 and 100 pings for each payload size, respectively), connecting the DUT at two different positions (first level-no PLC switching involved-, and second level-PLC switching-). PLC network has been configured to request acknowledge of any sent data packet.

The network is configured with a smart metering profile with $A R Q$, and carries metering traffic (short cycles are used, as described in [66]); a graphical representation of the PLC channel occupation is shown in Figure 7. The plot represents a ITU-T G.9904 superframe (time in X axis), where blue triangles (pointing up equal to uplink, and pointing down equal to downlink) are data packets, and red ones control traffic (the crosses show packets in the network that do not belong to the switching level that the $\mathrm{BN}$ can listen to-they are behind the device acting as a switch- but are somehow decoded even with invalid cyclic redundancy check (CRC)). Figure 8 shows the results for the delay of the ICMP pings, expressed as a histogram of the total percentage of packets below a specific delay. A worst case throughput for 32 byte-packets has been measured as $0.36 \mathrm{kbps}$, and $0.65 \mathrm{kbps}$ for 64 byte-packets. 


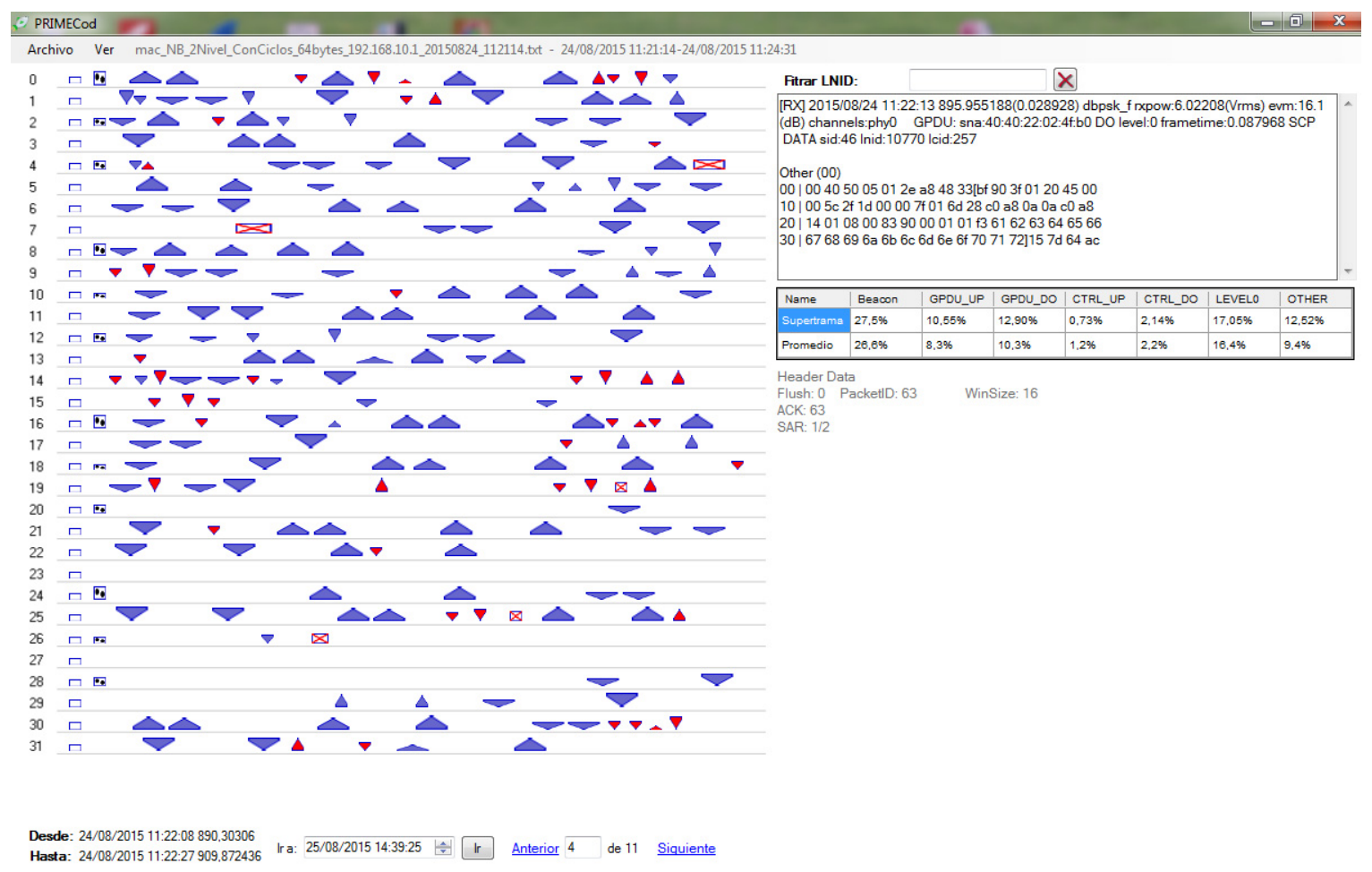

Figure 7. Traffic representation in PLC subnetwork under test.

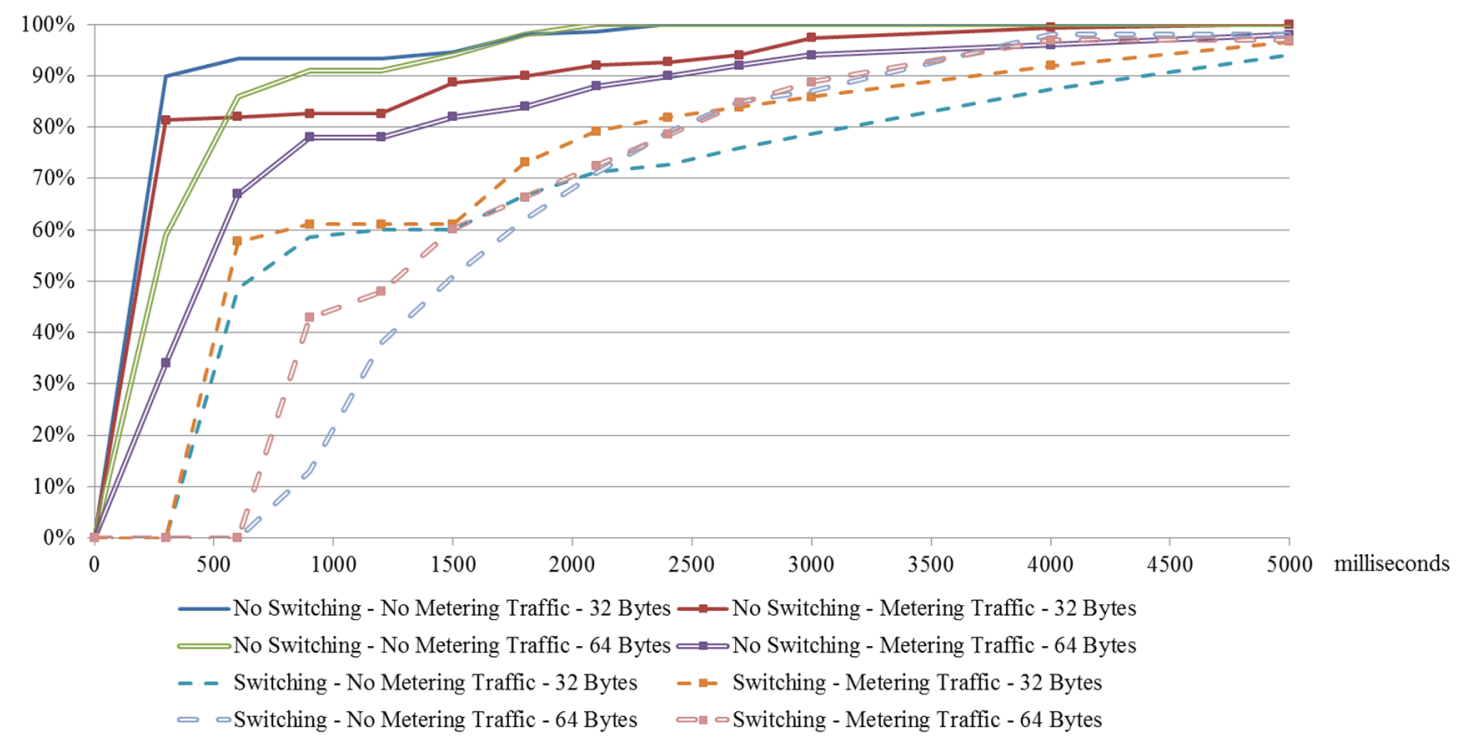

Figure 8. Internet control message protocol (ICMP) ping round-trip delay histogram.

\subsubsection{Tests Results Analysis and Conclusions}

The results presented for real field conditions demonstrate the feasibility of IP traffic transport over NB HDR PLC technologies in existing grid conditions, and in worst-case scenarios when smart metering traffic is present in the subnetwork. The results are coherent with the expected throughputs for "lossy" networks, and as such, should be handled for applications making use of them.

A further analysis of the test conditions shows that the results obtained can be significantly improved. The NB HDR PLC system implementation used for the tests is certified with the smart metering profile [64]. This means that standard features such as CFP (defined to ensure QoS for 
specific devices) and direct connections (designed to allow data traffic between peer devices without engaging the $\mathrm{BN}$ ) are not present. And some of the parameters present in the test (lowest throughput modulation, and 64 byte SAR mainly) could be improved. Thus, the tests have been performed under worst-case (or "best effort") conditions. With the introduction of the smart grid features, the adaptation of the conservative selections of smart metering applications, and the evolution of this technology into its next version [52], significantly improved results are expected.

\section{Powerline Communications Network Management}

A NMS, as it is defined in the IEC 60728-10, is a "software based system for controlling and supervising networks". A NMS for a NB HDR PLC telecommunication network must be able to manage the information on the performance of each PLC subnetwork to evaluate if it is working properly and how its performance can be improved.

In an ITU-T G.9904 system, the BN is the element controlling each subnetwork, and it has access to most of the real-time performance parameters of it. Out of the experience in the deployment of more than 30,000 ITU-T G.9904 subnetworks in Iberdrola Spain, the information needed to monitor the performance of the subnetworks can be categorized in the following groups:

- Subnetwork topology: number of SNs (terminals and switches), distribution of SNs within the subnetwork (at each subnetwork level), average availability of the SNs, detailed topology tree, etc.

- PLC channel occupation: number of bytes and occupied time of both CFP and shared contention period (SCP), classifying control and data traffic as well as downlink and uplink flow direction.

- Connection information (convergence layer): management connections, IEC 61334-4-32 connections, IP connections (Version 4 and 6), etc.

Apart from the monitoring capabilities, a NMS system needs to have the ability to follow the performance of the system in real-time. For this purpose, traffic capturing ("sniffing") capabilities must be added to be able to retrieve PLC packets exchanged by the BN.

\subsection{Simple Network Management Protocol}

The information should be available through a SNMP agent implementing a MIB, including the object identifiers (OIDs) object identifiers listed in Table 2.

Table 2. Object identifiers (OIDs) of the simple network management protocol (SNMP) management information base (MIB). Media access control: MAC.

\begin{tabular}{|c|c|c|}
\hline OID Group & OID Number & OID Name \\
\hline \multirow{10}{*}{ Subnetwork topology } & 1.3.6.1.4.1.x.1.2 & Number of terminals in the subnetwork \\
\hline & 1.3.6.1.4.1.x.1.4 & Number of beacons allocated \\
\hline & 1.3.6.1.4.1.x.1.5 & Number of switching levels \\
\hline & 1.3.6.1.4.1.x.1.6 & Number of switches per level \\
\hline & 1.3.6.1.4.1.x.1.8 & Number of promotions \\
\hline & 1.3.6.1.4.1.x.1.9 & Number of demotions \\
\hline & 1.3.6.1.4.1.x.1.10 & Number of registrations \\
\hline & 1.3.6.1.4.1.x.1.11 & Number of unregistrations \\
\hline & 1.3.6.1.4.1.x.1.12 & Successful alive base node (ALV)_B \\
\hline & 1.3.6.1.4.1.x.1.13 & Total ALV_B \\
\hline
\end{tabular}


Table 2. Cont.

\begin{tabular}{|c|c|c|}
\hline OID Group & OID Number & OID Name \\
\hline \multirow{10}{*}{$\begin{array}{l}\text { Topology tree (list of } \\
\text { registered nodes with the } \\
\text { following information of } \\
\text { each of them) }\end{array}$} & 1.3.6.1.4.1.x.2.MAC.1 & 1. MAC \\
\hline & 1.3.6.1.4.1.x.2.MAC.2 & 2. STATUS_Integer (0:Disconnected:1:Terminal:2:Switch:3:Base) \\
\hline & 1.3.6.1.4.1.x.2.MAC.3 & 3. LNID \\
\hline & 1.3.6.1.4.1.x.2.MAC. 4 & 4. Switch identifier (SID) (if not directly connected to the BN) \\
\hline & 1.3.6.1.4.1.x.2.MAC.5 & 5. Local switch identifier (LSID) (if it is a Switch)-Unsigned32 \\
\hline & 1.3.6.1.4.1.x.2.MAC.6 & 6. AVAILABILITY— $(1 / 10,000)$ \\
\hline & 1.3.6.1.4.1.x.2.MAC.7 & 7. DISCONNECTIONS \\
\hline & 1.3.6.1.4.1.x.2.MAC.8 & 8. Successful ALV_B \\
\hline & 1.3.6.1.4.1.x.2.MAC.9 & 9. Total ALV_B \\
\hline & 1.3.6.1.4.1.x.2.MAC.10 & 10. ALV_TIME \\
\hline \multirow{5}{*}{$\begin{array}{l}\text { Connection information } \\
\text { (convergence layer) }\end{array}$} & 1.3.6.1.4.1.x.3.1 & Total number of unicast active connections \\
\hline & 1.3.6.1.4.1.x.3.2 & Number of 4-32 connections \\
\hline & $1.3 \cdot 6.1 \cdot 4.1 \times 3.3$ & Number of management connections \\
\hline & 1.3.6.1.4.1.x.3.4 & Total number of multicast connections \\
\hline & 1.3.6.1.4.1.x.3.5 & Total number of devices registered in a multicast connection \\
\hline \multirow{15}{*}{ PLC channel occupation } & 1.3.6.1.4.1.x.4.1 & Number of packets in CFP \\
\hline & 1.3.6.1.4.1.x.4.2 & Time occupation (ms) of CFP \\
\hline & 1.3.6.1.4.1.x.4.3 & Number of packets in SCP \\
\hline & 1.3.6.1.4.1.x.4.4 & Time occupation (ms) of SCP \\
\hline & 1.3.6.1.4.1.x.4.5 & Number of error packets \\
\hline & 1.3.6.1.4.1.x.4.6 & Time occupation of error packets \\
\hline & 1.3.6.1.4.1.x.4.7 & Number of beacons received \\
\hline & 1.3.6.1.4.1.x.4.8 & Number of transmitted TX control packets \\
\hline & 1.3.6.1.4.1.x.4.9 & Number of received RX control packets \\
\hline & 1.3.6.1.4.1.x.4.10 & Time occupation of TX control packets (ms) \\
\hline & 1.3.6.1.4.1.x.4.11 & Time occupation of RX control packets (ms) \\
\hline & 1.3.6.1.4.1.x.4.12 & Number of TX data packets \\
\hline & 1.3.6.1.4.1.x.4.13 & Number of RX data packets \\
\hline & 1.3.6.1.4.1.x.4.14 & Time occupation of TX data packets (ms) \\
\hline & 1.3.6.1.4.1.x.4.15 & Time occupation of RX data packets (ms) \\
\hline
\end{tabular}

\subsection{Traffic Captures}

In order to analyze the traffic in the PLC subnetwork, an interface is to be defined in BNs to capture and send to the NMS every PLC packet data unit (PDU). This interface defines the TCP encapsulation for each PDU, including several fields in the header with information of the PDU (PDU type, origin and destination address, topology level, etc.) in order to facilitate the presentation of the information by the NMS to the operator. The interface defined is a custom simple binary protocol encoded in big endian that encapsulates messages as in Table 3.

Table 3. Traffic capture interface protocol.

\begin{tabular}{lcc}
\hline Length & Type & Payload \\
\hline 4 bytes & 1 byte & "Length" bytes \\
\hline
\end{tabular}

where:

- Length is the length of the payload. The messages are encoded sequentially and multiplexed using this length.

- Type is the type of the message. Currently there are just two types of messages: MAC PDU reception and transmission.

The payload will have the format in Table 4. 
Table 4. Traffic capture interface protocol. Payload format. Physical layer (PHY); packet data unit: PDU.

\begin{tabular}{cccccc}
\hline Time counter & Date and time & PHY Info & Len & PDU & Optional fields \\
\hline 4 bytes & 5 bytes & 2 bytes & 2 bytes & Len & - \\
\hline
\end{tabular}

- Time counter is a time counter of 10 microseconds that overflows every $12 \mathrm{~h}$.

- Date and Time are the number of seconds since 00:00 (midnight) 1 January 1970 GMT.

- PHY Info, as per Table 5.

Table 5. Traffic capture interface protocol. Payload physical layer (PHY) Info format.

\begin{tabular}{|c|c|c|}
\hline Bits & Type & Description \\
\hline 15 & Optional fields & 1 if optional fields are present, 0 otherwise. \\
\hline 14 & Interface & 1 if PLC, 0 other. If 0, SNR, power and encoding are not valid \\
\hline $10-13$ & Reserved & - \\
\hline \multirow{7}{*}{$7-9$} & \multirow{7}{*}{ Encoding } & The encoding of the PDU: \\
\hline & & 0 -D8PSK \\
\hline & & 1-DQPSK \\
\hline & & 2-D8PSK \\
\hline & & 4-DBPSK_CC \\
\hline & & 5-DQPSK_CC \\
\hline & & 6-D8PSK_CC \\
\hline \multirow{5}{*}{$4-6$} & \multirow{5}{*}{ SNR } & $\begin{array}{l}\text { Signal to noise ratio in which this message was received } \\
\text { (only reception) as defined in ITU-T G.9904 standard: }\end{array}$ \\
\hline & & $0: \leqslant 0 \mathrm{~dB}$ \\
\hline & & $1: \leqslant 3 \mathrm{~dB}$ \\
\hline & & $2: \leqslant 6 \mathrm{~dB}$ \\
\hline & & $7:>18 \mathrm{~dB}$ \\
\hline \multirow{10}{*}{$0-3$} & \multirow{10}{*}{ Power } & $\begin{array}{l}\text { For RX: reception power in which this messages was received } \\
\text { (only reception) as defined in ITU-T G.9904: }\end{array}$ \\
\hline & & $0: \geqslant 70 \mathrm{dBuV}$ \\
\hline & & $1: \geqslant 72 \mathrm{dBuV}$ \\
\hline & & $2: \geqslant 74 \mathrm{dBuV}$ \\
\hline & & 15: $>98 \mathrm{dBuV}$ \\
\hline & & For TX: \\
\hline & & 0: maximal output level (MOL) \\
\hline & & $1: \mathrm{MOL}-3 \mathrm{~dB}$ \\
\hline & & 2: $\mathrm{MOL}-6 \mathrm{~dB}$ \\
\hline & & $7: \mathrm{MOL}-21 \mathrm{~dB}$ \\
\hline
\end{tabular}

- Len is the length of the PDU payload.

- PDU is the MAC PDU buffer.

- Optional fields are optional information of each MAC PDU. This field can contain several fields with the format in Table 6.

Table 6. Traffic capture interface protocol. Payload Optional fields format.

\begin{tabular}{ccc}
\hline Field identifier & Field length & Data \\
\hline 1 byte & 1 byte & Field length \\
\hline
\end{tabular}




\subsection{Network Management Systems Use Case: Powerline Communications Subnetwork Monitoring}

The most basic subnetwork monitoring use-case for smart metering applications is based on the PLC topology, the packet count and the channel occupation control.

For this purpose, with the indications gathered from [18,67], the following information needs to be collected by the NMS from each subnetwork:

- Once per minute:

- Number of Terminals in the subnetwork-Unsigned 32.

- Number of Switches in the subnetwork-Unsigned 32.

- Average Availability—Unsigned 32 (as explained below).

- Once per hour:

- Number of TX Control Packets-Unsigned32.

- Number of RX Control Packets-Unsigned32.

- Time occupation of TX Control Packets (ms)-Unsigned32.

- Time occupation of RX Control Packets (ms)-Unsigned32.

- Number of TX Data Packets-Unsigned32.

- Number of RX Data Packets-Unsigned32.

- Time occupation of TX Data Packets (ms)-Unsigned32.

- Time occupation of RX Data Packets (ms) - Unsigned32.

- Once per day:

- List of registered nodes (Topology Tree).

- UpTime-Unsigned 32.

With this configuration of the use-case, the traffic expected per PLC subnetwork (i.e., domain of the $\mathrm{BN}$ ) to retrieve the information needed and considering an average subnetwork size of $130 \mathrm{SNs}$ is $0.813 \mathrm{MB}$ per day (optimizing SNMP requests through the use of "bulk" requests when possible). The volume of data to be stored in the database per each BN to comply with this configuration of the system, considering an average subnetwork size of 130 smart meters, is 24 kBytes per day.

Topology information is interesting to evaluate the behavior of the subnetwork in terms of stability. The average availability is calculated with the following formula:

$$
\text { Availability }_{1}=\frac{\sum_{i=1}^{n} \operatorname{Treg}_{i}}{n \times T_{\text {uptime }}}
$$

where:

- $T_{\text {reg: }}$ is the time that a particular node is registered in the subnetwork since the last reset.

- $n$ : is the number of different nodes registered in the subnetwork at least once since the last reset.

- $T_{\text {uptime: }}$ is the time since the last reset.

Channel occupation measures the use of the PLC subnetwork resources, obtaining patterns of use to detect any unexpected behavior which may damage the performance of the subnetwork.

The automatic analysis of the information received in the system, compared to the historical available information, will be used to represent trends and create alarms. Applying an intelligent algorithm to compare expected results with the actual situation of the subnetwork, and calibrating correctly the last results in order to avoid unnecessary alarms, the NMS will notify the operator alarms when a subnetwork is not performing according to its expected behavior, giving the operator the opportunity to manually retrieve additional OIDs and real-time detailed information (i.e., traffic 
capture interface), to analyze the situation and decide if it requires any remote or local, action or configuration.

\subsection{Powerline Communications Subnetwork Monitoring Tests}

Following the use-case of the previous section, several tests have been developed in different ITU-T G.9904 subnetworks, gathering the most important OIDs during a period of time. Some graphs and reports to check the performance have been generated.

Figure 9 shows the topology evolution of a subnetwork. It represents the number of terminals and switches registered in the subnetwork through time, giving a view of the stability of the subnetwork and showing possible events with a daily or hourly pattern.

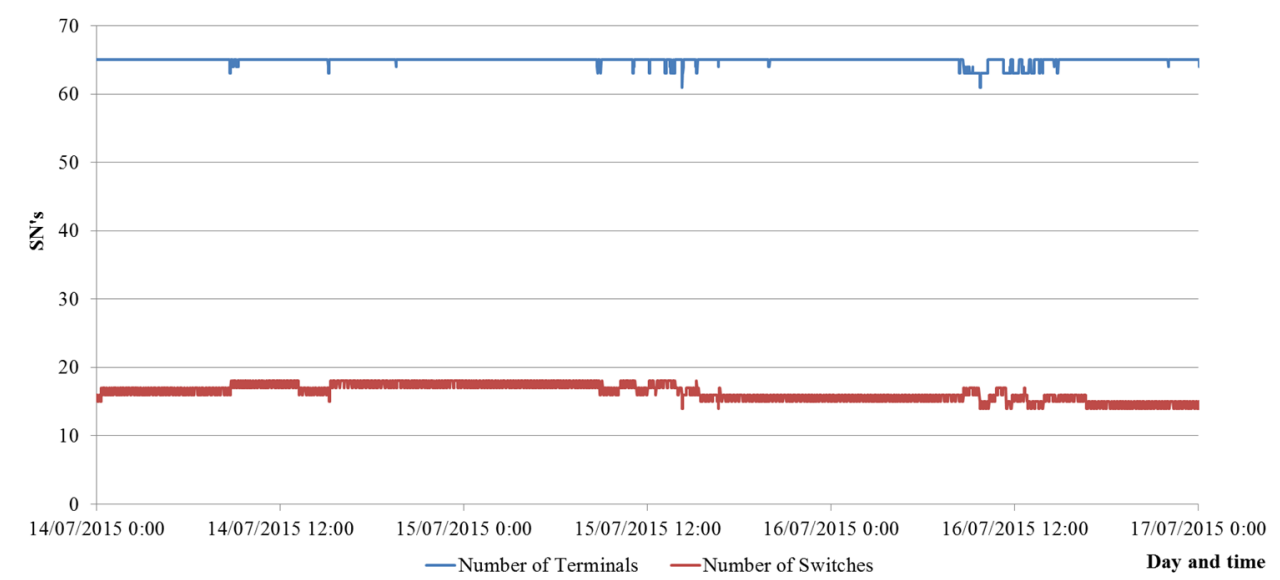

Figure 9. Topology SNs connected to the BN; terminals and switches) evolution of a PLC subnetwork.

Figure 10 shows the evolution of the number of control and data packets in the subnetwork. It gives information about the use of the communication resources provided by the subnetwork and the distribution of traffic along time.

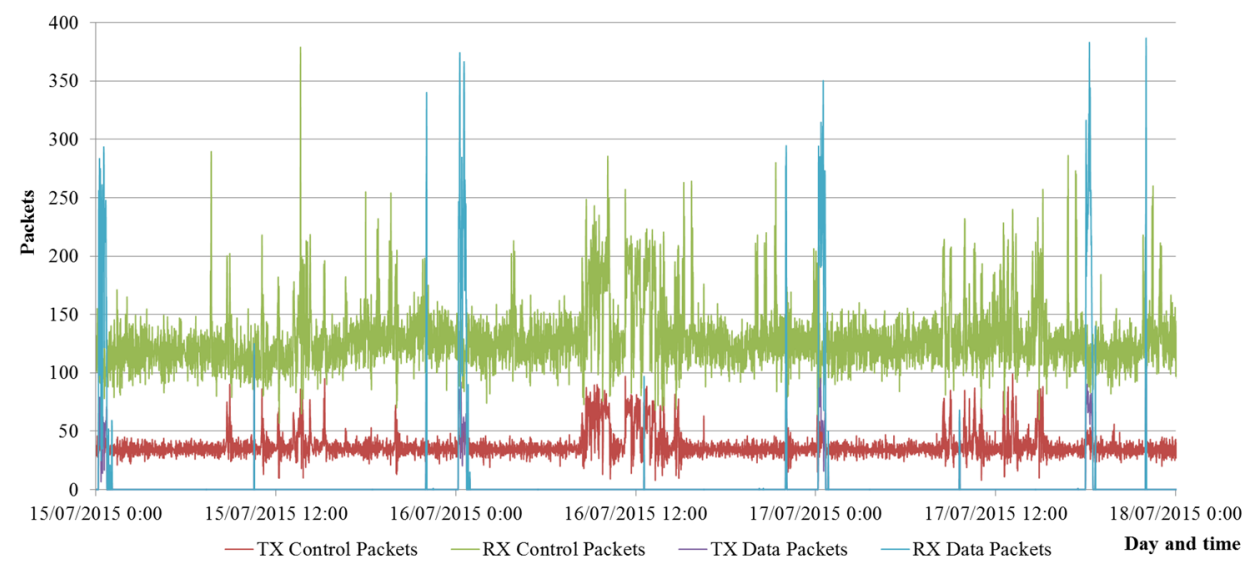

Figure 10. Control and data packets (transmitted -TX- and received -RX-) in a ITU-T G.9904 subnetwork.

Figure 11 shows the SCP occupation in the subnetwork. It represents the percentage of time in which the channel is occupied by any PDU in comparison with the duration of the SCP. Together with Figure 10, it gives information of the traffic in the subnetwork. 


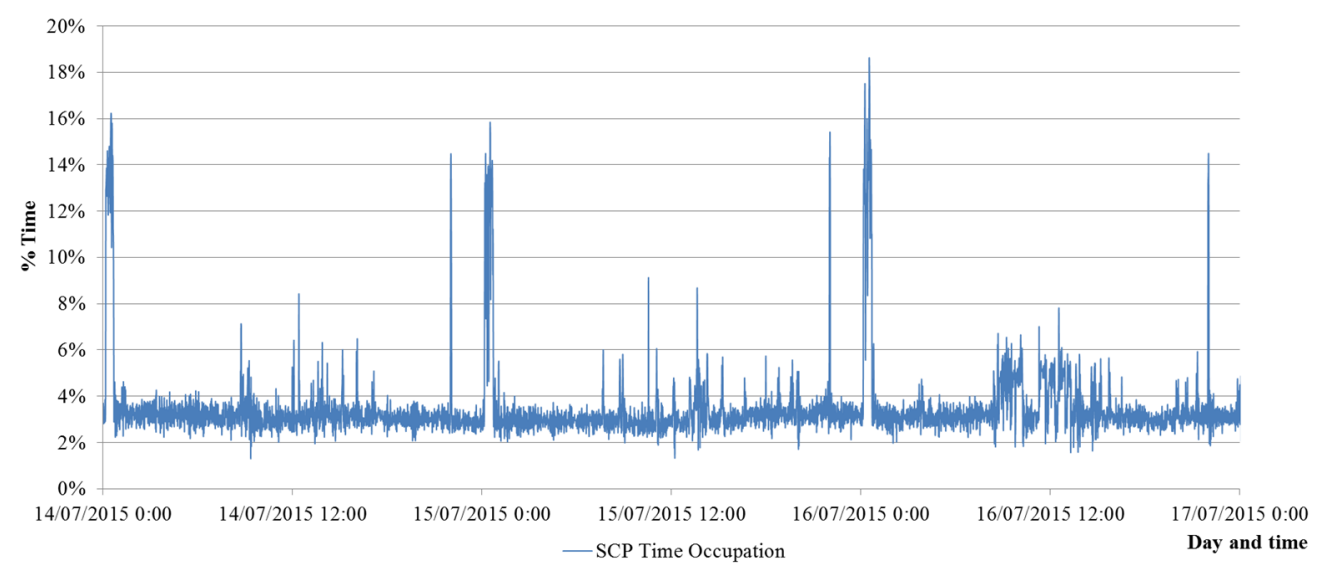

Figure 11. PLC channel occupation in a subnetwork.

Figure 12 contains an example of how the data can be analyzed to understand trends and study deviations. It shows the hourly channel occupation (number of transmitted data packets, received data packets, transmitted control packets and received control packets) of a PLC subnetwork with 100 SNs during one day. It is compared with the average traffic of this subnetwork in order to detect unexpected patterns.

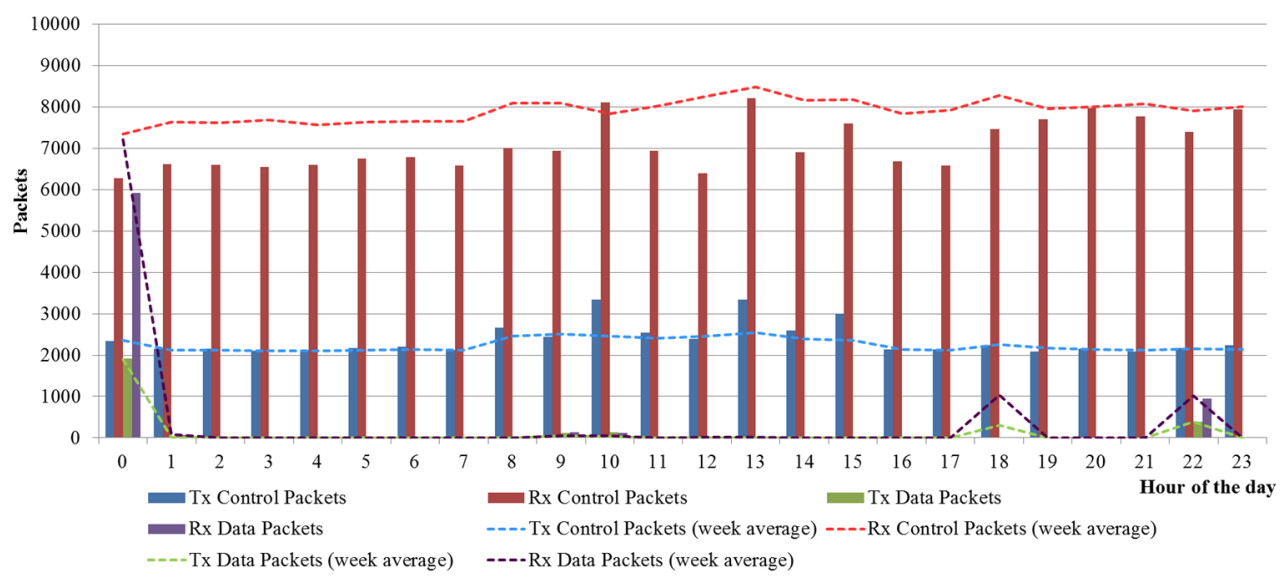

Figure 12. Graphical report of the number of data and control packets in the PLC subnetwork.

\section{Conclusions}

This paper has analyzed and addressed the challenges of the evolution of PLC-based smart metering networks towards the smart grid. The smart grid applications need support for controlled IP transport and PLC network management. This paper has analyzed the capacity of NB HDR PLC networks, and has shown with real tests the feasibility of such telecommunication capability over an ITU-T G.9904 implementation. This paper has also proposed a SNMP-based NMS system, and has shown how it can be used to offer an operational platform to manage NB HDR PLC networks.

As a conclusion, the evolution of NB HDR PLC-based smart metering systems into the smart grid is guaranteed. The operational procedures now in place for MV grids may also be implemented over PLC-deployed LV grids.

Acknowledgments: Supported by UPGRID project (European Commission Project 646531, Horizon 2020).

Author Contributions: All authors have coordinately worked in the different parts of the document.

Conflicts of Interest: The authors declare no conflict of interest. 


\section{References}

1. European Commission. Directive 2009/72/EC of the European Parliament and of the Council of 13 July 2009 Concerning Common Rules for the Internal Market in Electricity and Repealing Directive 2003/54/EC. European Commission: Brussels, Belgium, 2009.

2. European Commission. Benchmarking Smart Metering Deployment in the EU-27 with a Focus on Electricity; COM (2014) 356 Final 2014; European Commission: Brussels, Belgium, 2014.

3. Popa, M. Data collecting from smart meters in an Advanced Metering Infrastructure. In Proceedings of the 2011 15th IEEE International Conference on Intelligent Engineering Systems (INES), Poprad, Slovakia, 23-25 June 2011; pp. 137-142.

4. CEN-CENELEC-ETSI Smart Grid Coordination Group. CEN-CENELEC-ETSI Smart Grid Coordination Group-Sustainable Processes. European Commission: Brussels, Belgium, 2012.

5. Liotta, A.; Geelen, D.; van Kempen, G.; van Hoogstraten, F. A survey on networks for smart metering systems. Int. J. Pervasive Comput. Commun. 2012, 8, 23-52. [CrossRef]

6. Gungor, V.C.; Sahin, D.; Kocak, T.; Ergut, S.; Buccella, C.; Cecati, C.; Hancke, G.P. Smart Grid Technologies: Communication Technologies and Standards. IEEE Trans. Ind. Inform. 2011, 7, 529-539. [CrossRef]

7. Ferreira, H.C.; Lampe, L.; Newbury, J. Power Line Communications: Theory and Applications for Narrowband and Broadband Communications over Power Lines; Wiley: Chichester West Sussex, UK, 2010.

8. Ferreira, H.C.; Grové, H.M.; Hooijen, O.; Han-Vinck, A.J. Power Line Communication. In Wiley Encyclopedia of Electrical and Electronics Engineering; John Wiley \& Sons, Inc.: Chichester-West Sussex, UK, 2001.

9. Galli, S.; Scaglione, A.; Wang, Z. For the Grid and Through the Grid: The Role of Power Line Communications in the Smart Grid. Proc. IEEE 2011, 99, 998-1027. [CrossRef]

10. ITU-T G.9902 Recommendation. Narrowband Orthogonal Frequency Division Multiplexing Power Line Communication Transceivers for ITU-T G.hnem Networks; International Telecommunication Union: Geneva, Switzerland, 2012.

11. ITU-T G.9903 Recommendation. Narrowband Orthogonal Frequency Division Multiplexing Power Line Communication Transceivers for G3-PLC Networks; International Telecommunication Union: Geneva, Switzerland, 2014.

12. ITU-T G.9904 Recommendation. Narrowband Orthogonal Frequency Division Multiplexing Power Line Communication Transceivers for PRIME Networks; International Telecommunication Union: Geneva, Switzerland, 2012.

13. 1901.2-2013-IEEE Standard for Low-Frequency (Less than $500 \mathrm{kHz}$ ) Narrowband Power Line Communications for Smart Grid Applications; Institute of Electrical and Electronic Engineers: New York, NY, USA, 2013.

14. Papadopoulos, T.A.; Kaloudas, C.G.; Chrysochos, A.I.; Papagiannis, G.K. Application of Narrowband Power-Line Communication in Medium-Voltage Smart Distribution Grids. IEEE Trans. Power Deliv. 2013, 28, 981-988. [CrossRef]

15. Cataliotti, A.; Cosentino, V.; di Cara, D.; Tine, G. Measurement Issues for the Characterization of Medium Voltage Grids Communications. IEEE Trans. Instrum. Meas. 2013, 62, 2185-2196. [CrossRef]

16. Della Giustina, D.; Ferrari, P.; Flammini, A.; Rinaldi, S.; Sisinni, E. Automation of Distribution Grids with IEC 61850: A First Approach Using Broadband Power Line Communication. IEEE Trans. Instrum. Meas. 2013, 62, 2372-2383. [CrossRef]

17. Kabalci, E.; Kabalci, Y. A measurement and power line communication system design for renewable smart grids. Meas. Sci. Rev. 2013, 13, 248-252. [CrossRef]

18. Sendin, A.; Peña, I.; Angueira, P. Strategies for Power Line Communications Smart Metering Network Deployment. Energies 2014, 7, 2377-2420. [CrossRef]

19. Solaz, M.; Simon, J.; Sendin, A.; Andersson, L.; Maurer, M. High Availability solution for medium voltage BPL communication networks. In Proceedings of the 18th IEEE International Symposium on Power Line Communications and its Applications (ISPLC), Glasgow, UK, 30 March-2 April 2014; pp. 162-167.

20. Bush, S.F. Smart Grid: Communication-Enabled Intelligence for the Electric Power Grid; Wiley-IEEE Press: Chichester, West Sussex, UK, 2014.

21. Xiao, Y. Communication and Networking in Smart Grids; CRC Press: Boca Raton, FL, USA, 2012.

22. Goel, S.; Bush, S.F.; Bakken, D. IEEE Vision for Smart Grid Communications: 2030 and Beyond; Institute of Electrical and Electronic Engineers: New York, NY, USA, 2013. 
23. McDonald, J.D. Electric Power Substations Engineering, 3rd ed.; CRC Press: Boca Raton, FL, USA, 2012.

24. Zaballos, A.; Vallejo, A.; Majoral, M.; Selga, J.M. Survey and Performance Comparison of AMR over PLC Standards. IEEE Trans. Power Deliv. 2009, 24, 604-613. [CrossRef]

25. See, J.; Carr, W.; Collier, S.E. Real time distribution analysis for electric utilities. In Proceedings of the 2008 IEEE Rural Electric Power Conference, Charleston, SC, USA, 27-29 April 2008; pp. B5-B8.

26. Asimakopoulou, G.E.; Voumvoulakis, E.M.; Dimeas, A.L.; Hatziargyriou, N.D. Impact of large-scale integration of intelligent meters to the operation of the power system of crete. In Proceedings of the 2011 16th International Conference on Intelligent System Application to Power Systems (ISAP), Hersonissos, Greece, 25-28 September 2011; pp. 1-6.

27. Efthymiou, C.; Kalogridis, G. Smart Grid Privacy via Anonymization of Smart Metering Data. In Proceedings of the 2010 First IEEE International Conference on Smart Grid Communications (SmartGridComm), Gaithersburg, MD, USA, 4-6 October 2010; pp. 238-243.

28. Liu, E.; Chan, M.L.; Huang, C.W.; Wang, N.C.; Lu, C.N. Electricity grid operation and planning related benefits of advanced metering infrastructure. In Proceedings of the 5th International Conference on Critical Infrastructure (CRIS), Beijing, China, 20-22 September 2010; pp. 1-5.

29. Marron, L.; Osorio, X.; Llano, A.; Arzuaga, A.; Sendin, A. Low voltage feeder identification for smart grids with standard narrowband PLC smart meters. In Proceedings of the 17th IEEE International Symposium on Power Line Communications and Its Applications (ISPLC), Johannesburg, South Africa, 24-27 March 2013; pp. 120-125.

30. Santos, S; Llano, A.; Arzuaga, A.; Arzuaga, T.; Marron, L.; Zamalloa, M. Smart meters enable synchrophasor applications in distribution grids. In Proceedings of the 2012 CIGRE International Council on Large Electricity Systems, Paris, France, 26-31 August 2012.

31. Fang, X.; Misra, S.; Xue, G.; Yang, D. Smart Grid 2014: The New and Improved Power Grid: A Survey. IEEE Commun. Surv. Tutor. 2012, 14, 944-980. [CrossRef]

32. Sendin, A.; Urrutia, I.; Garai, M.; Arzuaga, T.; Uribe, N. Narrowband PLC for LV smart grid services, beyond Smart Metering. In Proceedings of the 18th IEEE International Symposium on Power Line Communications and its Applications (ISPLC), Glasgow, Scottland, 30 March-2 April 2014; pp. 168-172.

33. Smit, K. Experiences with PLC pilots in the Netherlands. In Proceedings of the 4th Workshop on Powerline Communications and its Applications in Networking and Smart Grid (WSPLC), Boppard, Germany, 22-24 September 2010.

34. Bumiller, G.; Lampe, L.; Hrasnica, H. Power line communication networks for large-scale control and automation systems. IEEE Commun. Mag. 2010, 48, 106-113. [CrossRef]

35. Akitomi, T.; Kikuchi, T. PLC Network Management System 2005. Technical Report. Available online: http://www.mitsubishielectric.com/company/rd/advance/pdf/vol109/vol109_tr4.pdf (accessed on 26 August 2015).

36. De Oliveira, D.N.; Vasques, T.L.; Vieira, F.H.T.; de Deus, G.A.; de Castro, M.S.; de Araújo, S.G.; de Souza, E.M.; Vieira, J.G.; Craveiro de Oliveira Junior, O.; Borges, A.; et al. A management system for PLC networks using SNMP Protocol. In Proceedings of the 2010 IEEE International Symposium on Power Line Communications and Its Applications (ISPLC), Rio de Janeiro, Brazil, 28-31 March 2010; pp. 78-83.

37. Kang, J.-M.; Park, C.-K.; Kim, E.-H.; Hong, J.W.-K.; Lim, Y.; Ju, S.; Choi, M.; Lee, B.; Hyun, D. Design and Implementation of Network Management System for Power Line Communication Network. In Proceedings of the IEEE International Symposium on Power Line Communications and Its Applications, Pisa, Italy, 26-28 March 2007; pp. 23-28.

38. Brown, P.A. Power line communications-past, present, and future. In Proceedings of the International Symposium on Power-line Communications and its Applications, Lancaster, UK, 30 March-1 April 1999; pp. 1-8.

39. Dostert, K. Powerline Communications, 1st ed.; Prentice Hall: Upper Saddle River, NJ, USA, 2001.

40. BS 6839-1:1987. Mains Signalling Equipment. Specification for Communication and Interference Limits and Measurements; British Standards Institution: Chiswick High Road, London, UK, 1987.

41. EN 50065-1:2011. Signalling on Low-Voltage Electrical Installations in the Frequency Range $3 \mathrm{kHz}$ to $1485 \mathrm{kHz}$. General Requirements, Frequency Bands and Electromagnetic Disturbances; European Commission: Brussels, Belgium, 2011. 
42. Electric Power Research Institute. Official Web Page. Available online: http://www.epri.com/Pages/ Default.aspx (accessed on 26 August 2015).

43. IEC 61334-4-41:1996. Distribution Automation Using Distribution Line Carrier Systems-Part 4: Data Communication Protocols—Section 41: Application Protocol-Distribution Line Message Specification; International Electrotechnical Commission: Geneva, Switzerland, 1996.

44. IEC 61334-4-42:1996. Distribution Automation Using Distribution Line Carrier Systems-Part 4: Data Communication Protocols—Section 42: Application Protocols-Application Layer; International Electrotechnical Commission: Geneva, Switzerland, 1996.

45. DIN 43863-3:1997-02. Elektrizitätszähler-Teil 3: Tarifgeräte als Zusatzeinrichtung zum Elektrizitätszähler; EDIS-Energie-Daten-Identifikations-System; Deutsches Institut für Normung: Berlin, Germany, 1997. (In German)

46. IEC 61334-5-1:2001. Distribution Automation Using Distribution Line Carrier Systems-Part 5-1: Lower Layer Profiles-The Spread Frequency Shift Keying (S-FSK) Profile; International Electrotechnical Commission: Geneva, Switzerland, 2001.

47. IEC TS 61334-5-2:1998. Distribution Automation Using Distribution Line Carrier Systems-Part 5-2: Lower Layer Profiles_Frequency Shift Keying (FSK) Profile; International Electrotechnical Commission: Geneva, Switzerland, 1998.

48. IEC TS 61334-5-3:2001. Distribution Automation Using Distribution Line Carrier Systems_Part 5-3: Lower Layer Profiles-Spread Spectrum Adaptive Wideband (SS-AW) Profile; International Electrotechnical Commission: Geneva, Switzerland, 2001.

49. IEC TS 61334-5-4:2001. Distribution Automation Using Distribution Line Carrier Systems-Part 5-4: Lower Layer Profiles-Multi-Carrier Modulation (MCM) Profile; International Electrotechnical Commission: Geneva, Switzerland, 2001.

50. IEC TS 61334-5-5:2001. Distribution Automation Using Distribution Line Carrier Systems-Part 5-5: Lower Layer Profiles-Spread Spectrum-Fast Frequency Hopping (SS-FFH) Profile; International Electrotechnical Commission: Geneva, Switzerland, 2001.

51. Bumiller, G.; Sauter, T.; Pratl, G.; Treytl, A. Secure and reliable wide-area power-line communication for soft-real-time applications within REMPLI. In Proceedings of the 2005 International Symposium on Power Line Communications and Its Applications, Vancouver, BC, Canada, 6-8 April 2005; pp. 57-60.

52. PRIME Alliance PRIME v1.4 White Paper October 2014. Available online: http:/ /www.prime-alliance.org/ wp-content/uploads/2014/10/whitePaperPrimeV1p4_final.pdf (accessed on 26 August 2015).

53. Woods, E. How Europe Is Reshaping the Smart Metering Market. Available online: http:/ / www.navigantresearch.com/blog/how-europe-is-reshaping-the-smart-metering-market (accessed on 26 August 2015).

54. Kim, I.H.; Kim, W.; Park, B.; Yoo, H. Channel measurements and field tests of narrowband power line communication over Korean underground LV power lines. In Proceedings of the 18th IEEE International Symposium on Power Line Communications and its Applications (ISPLC), Glasgow, UK, 30 March-2 April 2014; pp. 132-137.

55. Bauer, M.; Liu, W.; Dostert, K. Channel emulation of low-speed PLC transmission channels. In Proceedings of the IEEE International Symposium on Power Line Communications and Its Applications, Dresden, Germany, 29 March-1 April 2009; pp. 267-272.

56. ISO/IEC 12139-1:2009. Information Technology-Telecommunications and Information Exchange between Systems-Powerline Communication (PLC)—High Speed PLC Medium Access Control (MAC) and Physical Layer (PHY)_Part 1: General Requirements; International Organization for Standardization: Geneva, Switzerland, 2009.

57. Bettinsoli, L.; Napolitano, R.; Imposimato, C.; Giubbini, P. Impact of Non Intentional Disturbances on Distribution Line Communication. In Proceedings of the 23th CIRED International Conference on Electricity Distribution, Lyon, France, 15-18 June 2015.

58. Ronnberg, S.; Bollen, M. Measurements of primary and secondary emission in the supraharmonic frequency range 2-150 kHz. In Proceedings of the 23th CIRED International Conference on Electricity Distribution, Lyon, France, 15-18 June 2015. 
59. Leroi, C.; de Jaeger, E. Conducted Disturbances in the Frequency Range 2-150 kHz: Influence of the LV Distribution Grids. In Proceedings of the 23th CIRED International Conference on Electricity Distribution, Lyon, France, 15-18 June 2015.

60. ZIV PRIME Manager Tool, Metering Solutions. Available online: http://www.meteringsolutions.ziv.es/ documentacion/manuales/english/data_Sheets/ZIV_DISTRIBUTION_AUTOMATION_AMI_E_CG.pdf (accessed on 26 August 2015).

61. PBN PRIME Base Node, Metering Solutions. Available online: http://www.meteringsolutions.ziv.es/ documentacion/manuales/english/data_Sheets/ZIV_PRIME_BASE_NODE_SERVICE\%20NODE_E_CG.pdf (accessed on 26 August 2015).

62. IPERF Tool. XJPERF, Graphical Frontend for IPERF (Version jperf-2.0.2). Available online: https://code. google.com/p/xjperf/ (accessed on 26 August 2015).

63. Berganza, I.; Sendin, A.; Arzuaga, A.; Sharma, M.; Varadarajan, B. PRIME on-field deployment First summary of results and discussion. In Proceedings of the 2011 IEEE International Conference on Smart Grid Communications (SmartGridComm), Brussels, Belgium, 17-20 October 2011; pp. 297-302.

64. Meter Devices, PRIME Alliance. Available online: http://www.prime-alliance.org/?page_id=316 (accessed on 26 August 2015).

65. Approved Smart Meters, Iberdrola. Available online: https://www.iberdroladistribucionelectrica.com/ webibd/corporativa/iberdrola?IDPAG=ENSOCDISDISREDPREHOM (accessed on 26 August 2015).

66. Sendin, A.; Berganza, I.; Arzuaga, A.; Pulkkinen, A.; Kim, I.H. Performance results from 100,000+ PRIME smart meters deployment in Spain. In Proceedings of the 2012 IEEE Third International Conference on Smart Grid Communications (SmartGridComm), Tainan, Taiwan, 5-8 November 2012; pp. 145-150.

67. Sendin, A.; Berganza, I.; Arzuaga, A.; Osorio, X.; Urrutia, I.; Angueira, P. Enhanced Operation of Electricity Distribution Grids through Smart Metering PLC Network Monitoring, Analysis and Grid Conditioning. Energies 2013, 6, 539-556. [CrossRef]

(C) 2015 by the authors; licensee MDPI, Basel, Switzerland. This article is an open access article distributed under the terms and conditions of the Creative Commons by Attribution (CC-BY) license (http:/ / creativecommons.org/licenses/by/4.0/). 